\title{
NEAR-INFRARED CAMERA AND MULTI-OBJECT SPECTROMETER OBSERVATIONS OF THE HUBBLE DEEP FIELD: OBSERVATIONS, DATA REDUCTION, AND GALAXY PHOTOMETRY
}

\author{
RODGER I. THOMPSON \\ Steward Observatory, University of Arizona, Tucson, AZ 85721 \\ Lisa J. StorRie-Lombardi AND RAY J. WeymanN \\ Carnegie Observatories, 813 Santa Barbara Street, Pasadena, CA 91101 \\ AND \\ Marcia J. Rieke, Glenn Schneider, Elizabeth Stobie, and Dyer Lytle \\ Steward Observatory, University of Arizona, Tucson, AZ 85721 \\ Received 1998 August 24; accepted 1998 October 1
}

\begin{abstract}
This paper presents data obtained during the NICMOS Guaranteed Time Observations of a portion of the Hubble Deep Field. The data are in a catalog format similar to the publication of the original WFPC2 Hubble Deep Field program (Williams et al.). The catalog contains 342 objects in a $499^{\prime \prime} 1 \times 48.4$ subfield of the total observed field, 235 of which are considered coincident with objects in the WFPC2 catalog. The $3 \sigma$ signal-to-noise ratio level is at an aperture AB magnitude of approximately 28.8 at 1.6 $\mu \mathrm{m}$. The catalog sources, listed in order of right ascension, are selected to satisfy a limiting signal-tonoise ratio criterion of greater than or equal to 2.5. This introduces a few false detections into the catalog, and users should take careful note of the completeness and reliability levels for the catalog discussed in $\S \S 9$ and 10 . The catalog also contains a test parameter indicating the results of half-catalog tests and the degree of coincidence with the original WFPC2 catalog.
\end{abstract}

Key words: cosmology: observations - galaxies: fundamental parameters

\section{INTRODUCTION}

Deep observations with the Near-Infrared Camera and Multi-Object Spectrometer (NICMOS), devoted to understanding the nature of galaxy formation and evolution along with information on cosmological parameters, have always been an important aspect of the NICMOS Instrument Development Team (IDT) program since its inception in 1984. After the WFPC2 Hubble Deep Field (HDF) program in late 1995, it became obvious that observations at longer wavelengths would greatly enhance the value of the existing data in addition to satisfying the original intent of deep observations. The smaller field of view of the NICMOS instrument made it necessary to choose between deep observations of a portion of the HDF and a survey of the entire HDF at a brighter limiting magnitude. With the advent of the HDF, there existed a large disparity between the depth of the HDF and the depth of observations at near-infrared wavelengths (Connolly et al. 1997). Since the majority of objects that might be at redshifts unobservable with WFPC2 were expected to be relatively faint, the IDT decided to conduct a limited spatial survey to the faintest possible magnitude. The results of a NICMOS General Observer HDF survey program (Dickinson et al. 1997) will provide coverage over the entire HDF.

The NICMOS HDF program consists of 127 orbits out of a total of 553 orbits for the entire GTO program. Table 1 shows the distribution of orbits between the two filters and two grisms. An additional two orbits were dedicated to confirmation of guide star acquisition. Although the bulk of the orbits are dedicated to imaging, the large comoving volume for line observations available to the grisms is very appealing. The small number of grism orbits shown in Table 1 were intended as test cases to see if more GTO orbits should be transferred to this program. In order to expedite the delivery of image data to the community at large, we have not concentrated on the reduction of the grism data, and it is not presented in this publication. The grism data will be published in subsequent papers. As discussed below, the grism observations, however, significantly influenced the choice of the field for NICMOS imaging observations.

As with the Williams et al. (1996) paper, the purpose of this publication is a presentation of the data and analysis techniques rather than a discussion of the scientific content of the data. Future papers will discuss several implications of the new data. In the following we present the rationale for the observation methods, the methods for image production and source extraction, the catalog, and a discussion of the quality of the data in terms of signal-to-noise ratio, completeness, and reliability. Note that all magnitudes quoted in this paper are in the $\mathrm{AB}$ system.

\section{FIELD SELECTION}

The decision to devote part of the observational time to grism observations limited field choices to regions of the HDF that are not dominated by large bright foreground galaxies. The slitless dispersed spectra of these galaxies would overlap large areas of the field of view and reduce the number of spectral observations of fainter galaxies. Although some information on high-redshift objects was available at the time of field selection, no effort was made to bias the field position to include the largest number of highredshift sources.

The Space Telescope Science Institute decision to schedule the NICMOS HDF observations during the Camera 3 campaign in January of 1998 determined the acceptable range of roll orientations. This time period was not part of the continuous viewing zone (CVZ) opportunity period; 
TABLE 1

Basic Parameters of the NICMOS Hubble DeEp Field

\begin{tabular}{cccccccc}
\hline \hline $\begin{array}{c}\text { R.A. } \\
(\mathrm{J} 2000.0)\end{array}$ & $\begin{array}{c}\text { Decl. } \\
(\mathrm{J} 2000.0)\end{array}$ & $\begin{array}{c}\text { Orient } \\
(\mathrm{deg})\end{array}$ & $\begin{array}{c}\text { Total Field } \\
(\mathrm{arcsec})\end{array}$ & $\begin{array}{c}\text { F110W } \\
(\text { orbits })\end{array}$ & $\begin{array}{c}\text { F160W } \\
(\text { orbits })\end{array}$ & $\begin{array}{c}\text { G096 } \\
\text { (orbits) }\end{array}$ & $\begin{array}{c}\text { G141 } \\
\text { (orbits) }\end{array}$ \\
\hline $123645.129 \ldots \ldots$ & +621215.55 & 261.851 & $57.87 \times 61.34$ & 49 & 49 & 12 & 15 \\
\hline
\end{tabular}

NoTE.-Units of right ascension are hours, minutes, and seconds, and units of declination are degrees, arcminutes, and arcseconds.

however, it did offer a larger fraction of truly dark observing time. Given these constraints, a field located roughly at the center of the WFPC2 chip 4 field offered the best observational opportunities. The $\mathbf{J} 2000.0$ center position is $12^{\mathrm{h}} 36^{\mathrm{m}} 45^{\mathrm{s}} .129,+62^{\circ} 12^{\prime} 15^{\prime \prime} .55$. There is a relatively bright star $\left(\mathrm{AB}_{H}\right.$ of approximately $\left.22.1 \mathrm{mag}\right)$ near the center that provides an excellent fiducial location for the grism observations. The final orient of 261.851 is the result of fine tuning to obtain the best possible guide star orientation.

\section{FILTER AND CAMERA SELECTION}

\subsection{Camera Selection}

All of the NICMOS HDF data in this paper are from Camera 3. The wide field format of Camera 3 made it the obvious choice for HDF observations. The campaign also utilized Cameras 1 and 2 with the same integration parameters as Camera 3, but they were not in focus during operation. Parallel observation of these cameras with Camera 3 prevented the occurrence of the faint artifacts, termed bars, which occur when the autoflush and imaging output timing patterns overlap. Similar integration parameters for all cameras prevents the parallel cameras from defaulting into the autoflush pattern. The data from these cameras may be useful for background characterization but are not analyzed in this publication.

\subsection{Filter Selection}

The observations employed two imaging filters for Camera 3, F110W and F160W centered at 1.1 and $1.6 \mu \mathrm{m}$, respectively. By careful design, the F160W spans the lowest background spectral region available to NICMOS. This is the minimum between the scattered zodiacal emission that decreases with wavelength and the thermal emission from the warm Hubble Space Telescope (HST) mirrors that

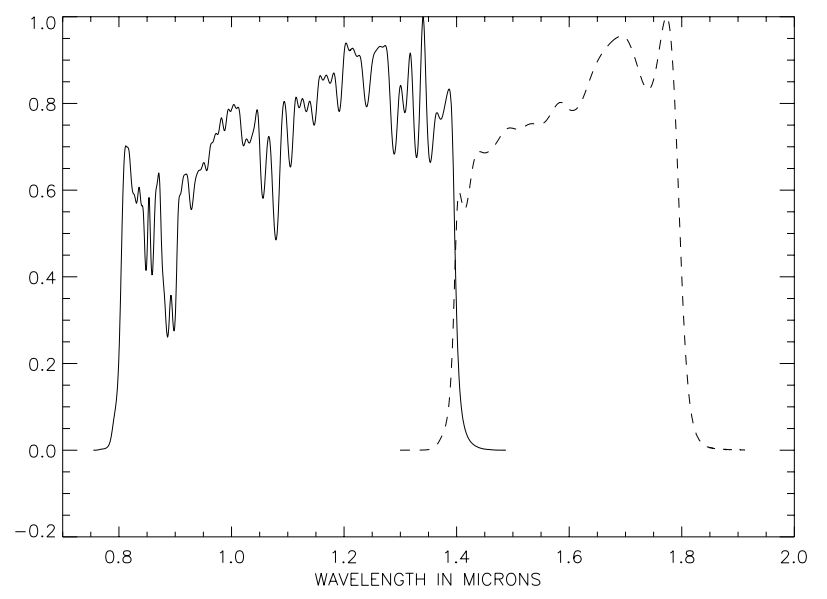

FIG. 1.-Normalized total filter response functions for the NICMOS F160W and F110W filters. These response functions include all of the color-dependent terms including the detector quantum efficiency. increases with wavelength. Both of these emissions were lower than expected prior to the HDF observations. The second filter covers a shorter wavelength over a rather broad bandwidth. This filter provides a second color between the F814W WFPC2 filter and the NICMOS F160W filter. The addition of this filter provides an important discriminator between high-redshift star-forming galaxies, which will have blue infrared colors, and lower redshift galaxies with large amounts of dust extinction, which will have red infrared colors. Although very useful for all objects, the extra filter is particularly important for objects detected only in the NICMOS bands. Figure 1 displays the normalized detectivity for the NICMOS filters. These plots include all of the color-dependent terms of the detectivity, including the detector quantum efficiency. The rapidly changing indices of refraction for most optical coating materials in the $1 \mu \mathrm{m}$ region account for the complicated shape of the F110W filter.

\section{OBSERVATIONAL STRATEGY}

The preflight decision to devote 49 orbits to each imaging filter and 27 orbits to grism spectroscopy set the parameters for the observing strategy. The 49 orbits are based on a $7 \times 7$ grid of positions, and the grism orbits employ three different roll angle positions to help remove confusion from overlapping spectra. There are three separate integrations in each orbit to ensure that any problems encountered did not necessarily compromise all of the data during the orbit. Before the observations it appeared that the sky background would be the dominant noise source after about $900 \mathrm{~s}$ of integration. The lower than expected sky brightness, however, reduced the sky noise below the read noise for this integration time.

\subsection{Detector Sampling Sequence}

Since there are no bright sources in the HDF, the logarithmic NICMOS sampling sequences, designed to handle high dynamic range images, are inappropriate. Those sequences add amplifier glow noise with several short time integrations near the beginning of the exposures. Good cosmic-ray rejection, on the other hand, requires a sufficient number of samples to establish an accurate signal after any cosmic-ray-affected samples are rejected. The HDF integrations utilize the SPARS64 sampling sequence that has evenly spaced $64 \mathrm{~s}$ sample times after the first three short integration samples. The integrations have a NSAMP value of 17 , which produces a total integration time of $896 \mathrm{~s}$. Three of these integrations fill an orbit. The total number of integrations in each filter is then 147 integrations of $896 \mathrm{~s}$ in each filter for a total of $1.31712 \times 10^{5} \mathrm{~s}$ or $36.5 \mathrm{hr}$ of observing time per filter.

\subsection{Field Coverage}

Several factors influenced the choice of field size. The basic purpose of the NICMOS HDF program is deep 
observations. This requirement favors very small or no dithers away from the field center. Accurate background subtraction requires many offsets large enough to ensure that most pixels have the majority of their observations off of detectable objects. Spatial cosmic-ray rejection and image resolution enhancement require at least 1 pixel and fractional pixel offsets, respectively.

The pattern of observation positions on the sky is a combination of a small three-point dither pattern during each orbit and a larger $7 \times 7$ raster pattern that covered the 49 orbits. The dither pattern is a three-position spiral dither with a step size of 0 " 408 , roughly 2 Camera 3 pixels. The $x$ and $y$-spacings of the orbit-to-orbit raster are 0.918 and 1.523, respectively, which are 4.5 and 7.5 Camera 3 pixels. The interorbit moves were accomplished with target offsets from the original center position. The basic purpose of the raster was to move the field of view sufficiently that any single pixel had the majority of its integrations with no observable source present.

\subsection{Pointing Accuracy}

Owing to the paucity of bright stars in the HDF region and the roll constraints during the observational time period, we were not able to utilize two FGS guide stars. This situation led to the possibility of roll errors in position about the location of the single guide star. Real time frequent updates of the gyro bias levels by HST Missions Operations Support Engineering Systems (MOSES) mitigated this problem. Data provided by the MOSES team (C. Conner 1998, private communication from the Missions Operations Support Engineering Systems) indicated that the positional errors for all orbits used in this paper were less than 0.2 NICMOS Camera 3 pixels. Subsequent analysis discussed in $\S 5.3$ confirmed this data. Our absolute positions assume that the central star in our field (WFPC2 4-454) has the position stated in the published catalog (Williams et al. 1996).

\section{DATA REDUCTION}

Data reduction procedures utilized the Interactive Data Language (IDL) software environment for most of the basic data analysis. KFOCAS (K. Adelberger \& C. C. Steidel 1996, private communication to STScI), a derivative of FOCAS (Jarvis \& Tyson 1981; Valdes 1982) provided the source detections listed in the catalog. In order to provide a cross-check on the images and catalog presented in this paper, we have deliberately reduced and analyzed the data in two separate and independent ways. Specifically, in addition to the IDL and KFOCAS procedures, we utilized an independent IRAF-based image processing algorithm and an alternative source extraction program, SExtractor (Bertin \& Arnouts 1996). The IDL and KFOCAS reduction procedures are described in detail as they produced the bulk of the information on the sources listed in the catalog. Descriptions of the IRAF and SExtractor reductions are provided when they differ substantially from the IDL and KFOCAS reductions.

\subsection{IDL Image Reduction}

Each 896 s SPARS64 integration produced an individual image. A set of 55 SPARS64 dark integrations of the same duration as the HDF exposures provided the required dark frames for the analysis. These dark exposures occurred just prior to and coincident with the HDF exposures. The data reduction procedures produce a completely processed image for each integration. Section 5.3 describes the combination of the images into the final mosaic.

\subsubsection{Dark Frames}

Dark frame reductions begin with the division of each 17 sample SPARS64 darks into 16 first differences. A first difference is simply the difference between a readout and the previous readout. The first differences are then combined via a sigma clipping mean to produce a final superframe that is free of cosmic rays to the $3 \sigma$ level of the 55 combined observations. Although for most observations a simple median of first differences would suffice, observations at the sensitivity level of the HDF required sigma clipped means to avoid digitization noise. The average Camera 3 dark current is 0.2 electrons $\mathrm{s}^{-1}$. In each $64 \mathrm{~s}$ first difference, about 12.8 electrons accumulate. The detector gain for Camera 3 is 6.5 electrons per ADU for an average of 2 ADUs per first difference. If medians are taken of these observations, there would still be $50 \%$ noise even for an infinite number of integrations. The first differences are then recombined to produce a ramp dark for subtraction from the imaging integrations. The ramp dark is a sequence of summations ranging from just the initial first difference, the initial and second first difference, to the total of all of the first differences.

\subsubsection{Image Frames}

Analysis of the image integrations starts with the production of a set of 16 ramp readouts for the 17 samples from each SPARS64 integration. Subtraction of the superdark ramp from each integration produces a set of dark currentcorrected but not sky-corrected integrations. A set of standard linearity corrections is next applied to pixels that have exceeded the linear signal response region but have not saturated. In practice only bad "hot" pixels receive a correction owing to the low signal levels in the HDF observations.

After correction for linearity, the integrations are corrected for cosmic rays by fitting a linear function to the ramp values. The slope of this function is the signal rate in ADU per second. Cosmic rays produce an instantaneous discontinuity in the signal function. Subtraction of the fitted function from the signal produces an output that has a distinctive $S$ shape if a cosmic ray is present. In one readout the difference between the fit and the signal transitions from negative to positive. Detection of a transition greater than expected from noise indicates the presence of a cosmic ray. The offending first difference is then removed from the ramp, and a new fit is calculated. The new fit is again checked for cosmic rays, and detected cosmic rays are removed in a similar matter. Any fits still beyond the expected noise are declared bad and flagged as bad. If the cosmic ray produces saturation, only the readouts before saturation are used in the final fit. All detected cosmic rays are recorded in the data-quality image extension. This procedure is unique to the NICMOS instrument on HST owing to the ability to read out nondestructively the detector during the integration. Further cosmic-ray removal can occur if necessary during the image mosaic construction in the standard manner.

Before the flat field can be applied, all of the quadrant biases in the individual images must be removed. If there is a bias level in the image, the flat-field function will produce 
variations in the bias that will remain in the data. The removal procedure can be iterative, but in practice one iteration is sufficient. The first step produces a median of all of the cosmic-ray-corrected images. This median is then subtracted from each individual image. If there are no quadrant biases, the median value in each detector quadrant should be zero since the dark current and sky are removed by the subtraction. The sources do not dominate the image, so they contribute very little to the median. The second step measures the median of each quadrant in each image and records the value. The final step then subtracts these quadrant bias levels from each cosmic-ray-corrected image. The flat-field correction was then applied to each image.

The bad pixels are marked from a bad pixel mask determined from the previous observing history with the NICMOS Camera 3. The bad pixel mask contains both low response pixels and hot pixels defined as pixels with an excessive dark current. In each image the bad pixels were replaced by the median of the total image. The drizzle mosaic process does not use pixels marked as bad in the final image construction.

The median of all of the final individual images determines the level of the sky emission. The raster and dither of the large number of images reduces the source contribution of the median to a value less than the expected noise level. Inspection of the median image did not reveal any source contributions. Subtraction of this sky level from each image completes the analysis of the individual images. The median sky level in the F160W filter is 0.55 electrons $\mathrm{s}^{-1}$, which is lower than the original estimates prior to the observations. This is not surprising as one of the selection criteria for the HDF was a low zodiacal background.

\subsection{IRAF Image Reduction}

For comparison with the IDL procedures, images were reduced in an independent pipeline using NICRED 1.5 (McLeod 1997; Lehar et al. 1999) and modified IRAF scripts developed to reduce Camera 3 images taken in parallel mode (Yan et al. 1998). Two median sky-dark frames were produced, one from the first exposure in each orbit and one from combining the second and third exposures in each orbit, to minimize the effect of any pedestal in the first exposure. These were used as dark frames along with the same flats utilized in the IDL reductions. These flats, observed on 1997 December 23, are identical to the flats used in the IDL reduction. The residual bias levels in the individual quadrants were removed by fitting a Gaussian to a histogram of the pixels in each quadran and subtracting the peak value. A new bad pixel mask was created from the exposures. The images were inspected, and any remaining cosmic-ray hits or satellite trails were individually masked.

\subsection{Mosaic Techniques}

Both of the data reduction procedures utilize modified versions of the drizzle software developed for the reduction of the WFPC2 HDF images. The drizzled pixel size in each case is $\sim 0$ " 1 , one-half of the original NICMOS Camera 3 pixel size. The drizzle parameter PIXFRACT is 0.6 in the drizzling of the IDL reductions, while it was set to 0.65 in the drizzling of the IRAF results.

\subsubsection{IDL Image Mosaic}

The first task of mosaic production is an accurate determination of the relative offsets between the individual inte- grations. We compared offset information from the world coordinates in the header files, shifts computed from the IRAF/STSDAS Dither package, offsets from the IRAF imcentroid package of five individual objects in the field, and finally individual inspection via interactive IDL tools. The NICMOS geometric distortions have been determined to be negligible so no geometric distortion corrections were made.

In general, the agreement among the four methods was quite good. For the F160W images the discrepancy between the world coordinate shifts and the IRAF-generated shifts fell between -0.2 and 0.4 pixels in $x$ with a mean of 0.15 $(\mathrm{rms}=0.03)$ and a range of -0.5 to 0.2 pixels in $y$ with a mean of $-0.15 \quad(\mathrm{rms}=0.03)$. The internal difference between the shifts determined by IRAF procedures averaged -0.02 pixels.

For the F110W filter the difference between the world coordinate shifts and those determined by IRAF procedures varied from -2.0 to 0.7 pixels in $x$ and -0.4 and 2.7 pixels in $y$ with means of $0.1(\mathrm{rms}=0.14)$ and $0.7(\mathrm{rms}=0.36)$, respectively. The mean internal difference between the IRAF procedures was -0.12 for $x$ and 0.04 in $y$. The large excursions of 2.0 and 2.7 pixels in $x$ and $y$ were seen in only two images. The IRAF Dither and imcentroid positions agreed to 0.1 pixels in these images. Visual inspection of the images confirmed the IRAF positions. In both the F160W and the F110W filters the rotation angle varied by less than 0.005 owing to the excellent effort of the MOSES group in limiting the roll during the single guide star observations.

The data were drizzled using Drizzle Version 1.2 February, 1998 (Fruchter \& Hook 1997) with image offsets derived from the mean of the IRAF procedures since in cases in which the IRAF positions differed from the world coordinates, interactive inspection via the IDL tools confirmed the IRAF positions. As discussed above, no geometric distortion correction or image rotation was required. High cosmic-ray persistence noise levels after transit of the South Atlantic Anomaly (SAA) required removal of 28 F160W integrations and $36 \mathrm{~F} 110 \mathrm{~W}$ integrations from the final mosaic image. A comparison of a straight combination of the drizzled frames and a combination averaged with $3 \sigma$ clipping showed no differences, which indicates that the IDL cosmic-ray removal techniques were effective.

\subsubsection{IRAF Image Mosaic}

The IRAF reduction images were drizzled (Drizzle Version 1.1; Fruchter et al. 1997) with offsets determined from the centroid of the central star (NICMOS 249) in each frame. No rotation or geometric distortion corrections were necessary. Owing to persistence of cosmic rays encountered in SAA passages, 25 F160W images and 37 F110W images were removed from the final mosaic. Which frames to remove was determined independently by inspection, which leads to the slight difference from the number not included in the IDL image mosaic. The drizzled frames were averaged with $3 \sigma$ clipping to remove any residual low-level cosmic rays.

\section{THE IMAGES}

Figures 2 and 3 show the F110W and F160W images, respectively, produced by the IDL image reduction and drizzle procedures described in the preceding section. The raster pattern of observations produces much lower signalto-noise ratio areas in the image at the edges at which the 


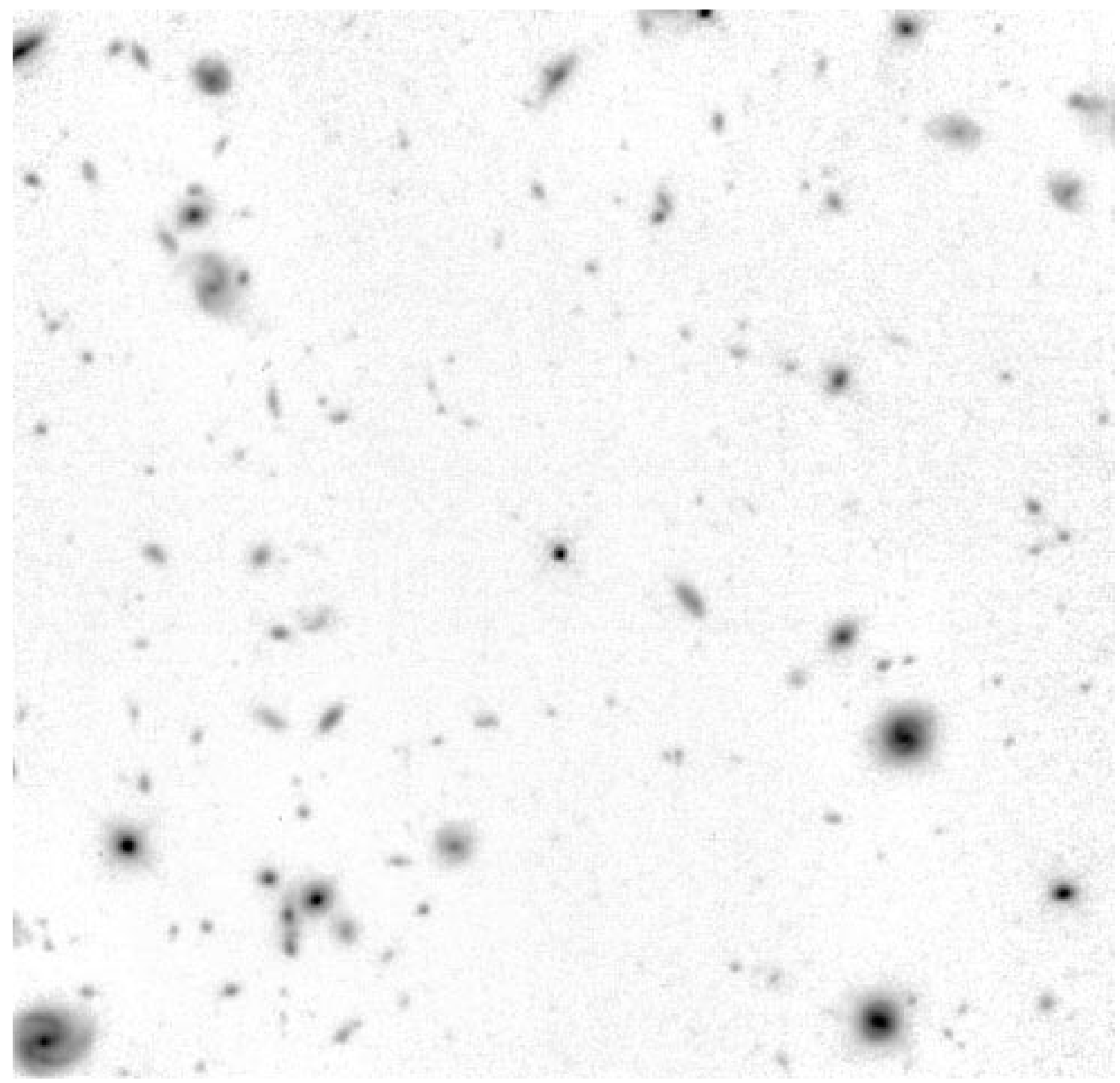

FIG. 2.-F160W image

number of overlapping integrations are greatly reduced. These areas are deleted from the image even though many strong sources are evident in these regions. The area covered by the images is a 49 ". $19 \times 48$ ×.53 (481 $\times 476$ pixels) rectangle. Figure 4 is a color composite of the two infrared images and the F606W WFPC2 image. The WFPC2 image has been rotated and resampled to fit the orientation and pixel size of the NICMOS images. The red, green, and blue colors represent the F160W, F110W, and F606W intensities. As with the original WFPC2 color image, the stretch and color curves have been manipulated to show faint objects while preserving the detail of features in the brighter objects. This image should not be used for quantitative purposes. Figures 2 and 3 are also stretched to show the best range of features. The very high dynamic range of the image can not be displayed in a linear intensity image.

\section{SOURCE DETECTION AND PHOTOMETRY}

Since the original WFPC2 HDF catalog (Williams et al. 1996) utilized KFOCAS to generate its listings, our primary catalog listings also utilize KFOCAS to provide consistency. We also provide a description of the SExtractor source extraction process. The main difference between KFOCAS and FOCAS is the utilization of a supplemental image by KFOCAS that specifies the relative detectivity at each point in the image. This is important for the NICMOS HDF images where there are significant variations of quantum efficiency and total integration time over the image area.

7.1. Estimation of the Input Sigma for KFOCAS

KFOCAS uses a constant $1 \sigma$ level that is either deter- 


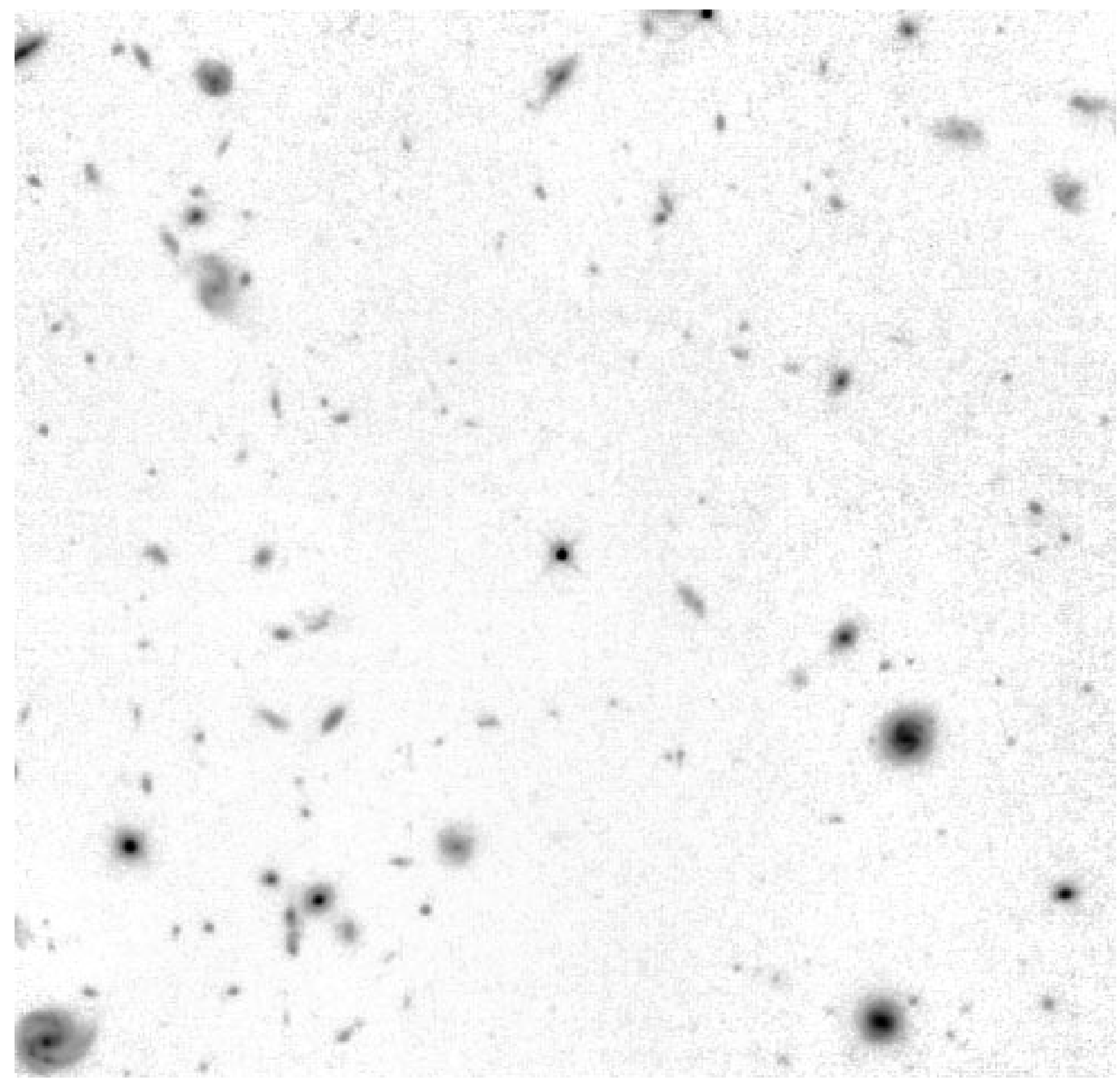

FIG. 3.-F110W image

mined from the first few lines of the image or is input manually by the user. Since the first few lines of the NICMOS image have much lower signal-to-noise ratio than rest of the image, we estimated the $1 \sigma$ value manually from a histogram of the signal levels in all of the pixels. Figure 5 gives the histogram of the pixel values in the two dithered images shown in Figures 2 and 3. Only the pixels from the area covered by the images are used in this histogram. The histogram peaks at zero signal as expected for sky-subtracted observations. The long extensions of the histograms toward positive values owing to the sources in the field are cut off in this figure. The flat-fielding process multiplies the true noise value in the image by the value of the flat field. This process raises the noise level in low quantum efficiency areas and lowers it in high-efficiency areas. Since the median efficiency of the area is set to 1, this process should not appreciably alter the width of the curve.

As learned in the production of the WFPC2 images, the drizzling process produces a correlated image and hence correlated noise (Williams et al. 1996; A. Fruchter 1998, private communication). There is approximately a factor of 2 reduction in the apparent noise as a result of the drizzle process for a factor of 2 reduction in linear pixel size. The numbers given in Figure 5 should therefore be multiplied by a factor of 2 to determine the true $1 \sigma$ value of the noise. This gives the noise figures of $1.22 \times 10^{-9} \mathrm{Jy}$ for the F160W filter and $1.54 \times 10^{-9} \mathrm{Jy}$ for the F110W filter. These are the powers that produce a signal equal to a $1 \sigma$ noise in a single pixel. Use of these levels resulted in KFOCAS missing a large number of real sources easily identified by eye. We 


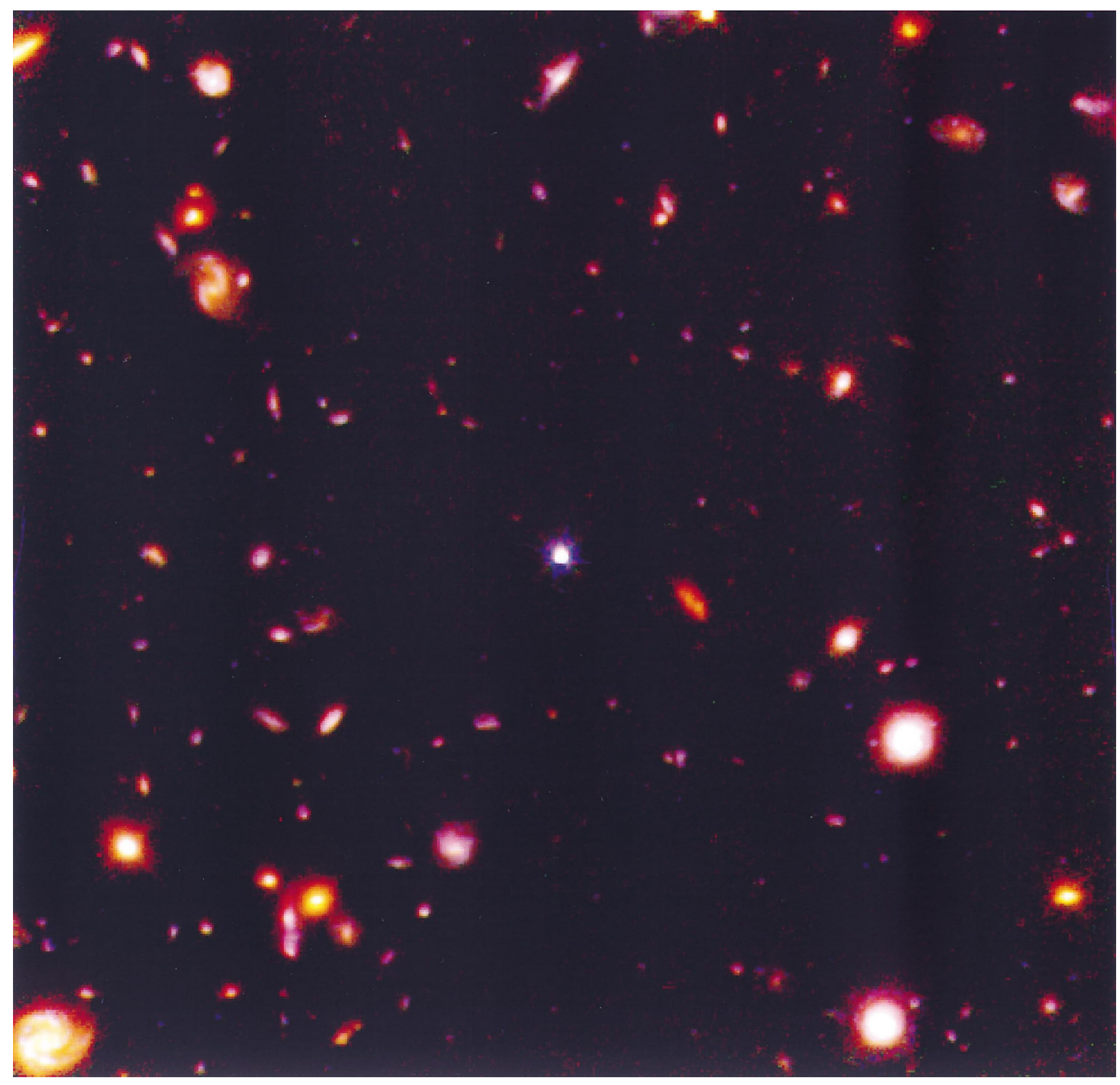

FIG. 4.-Composite color image

therefore dropped the $1 \sigma$ estimates to a very low value of $5.5 \times 10^{-10} \mathrm{Jy}$ or $2.0 \times 10^{-4}$ ADUs s ${ }^{-1}$ for the F160W filter and $3.5 \times 10^{-10} \mathrm{Jy}$ or $2.3 \times 10^{-4} \mathrm{ADUs} \mathrm{s}^{-1}$ for the F110W filter. The number of $\sigma$ for the detection limit was then varied until all known real sources were detected without excessive over selection. It is of course the product of the chosen $1 \sigma$ noise level and the number of sigma for detection parameter that determines the signal value a pixel must have to be considered a potential source. A known real source is an object easily seen by eye in the NICMOS images that is exactly coincident with an observed source in the WFPC2 HDF images. A more rigorous discussion of the completeness and reliability of the selected sources occurs in $\S \S 9$ and 10 . The catalog listings are limited to sources with signal-to-noise ratios that exceed or equal 2.5. This discards some sources that by many tests appear to be real but eliminates a large number of sources that have a significant chance of being false.

\subsection{KFOCAS Reduction}

We prepared the drizzled images for the KFOCAS procedure by multiplying the signal in ADUs $s^{-1}$ by $10^{5}$ and subtracting the minimum value, a negative number, from the multiplied image. This produced an image that had no negative values and in which all of the significant values were well represented in the integer arithmetic used by KFOCAS. The zero-point magnitudes of the modified images are 35.3 for the F160W image and 35.186 for the F110W image. The source extraction utilized the standard KFOCAS procedures of the series KDETECT, KSKY, KEVALUATE, KSPLIT, and RESOLUTION. Our drizzled pixels have 6.25 times the area of the WFPC2 drizzled pixels; therefore, we set the minimum area for detection in pixels to 2 in order to avoid missing very compact galaxies. The parameters for the KFOCAS reduction are listed in Table 2. The point-spread function (PSF) matrix for 

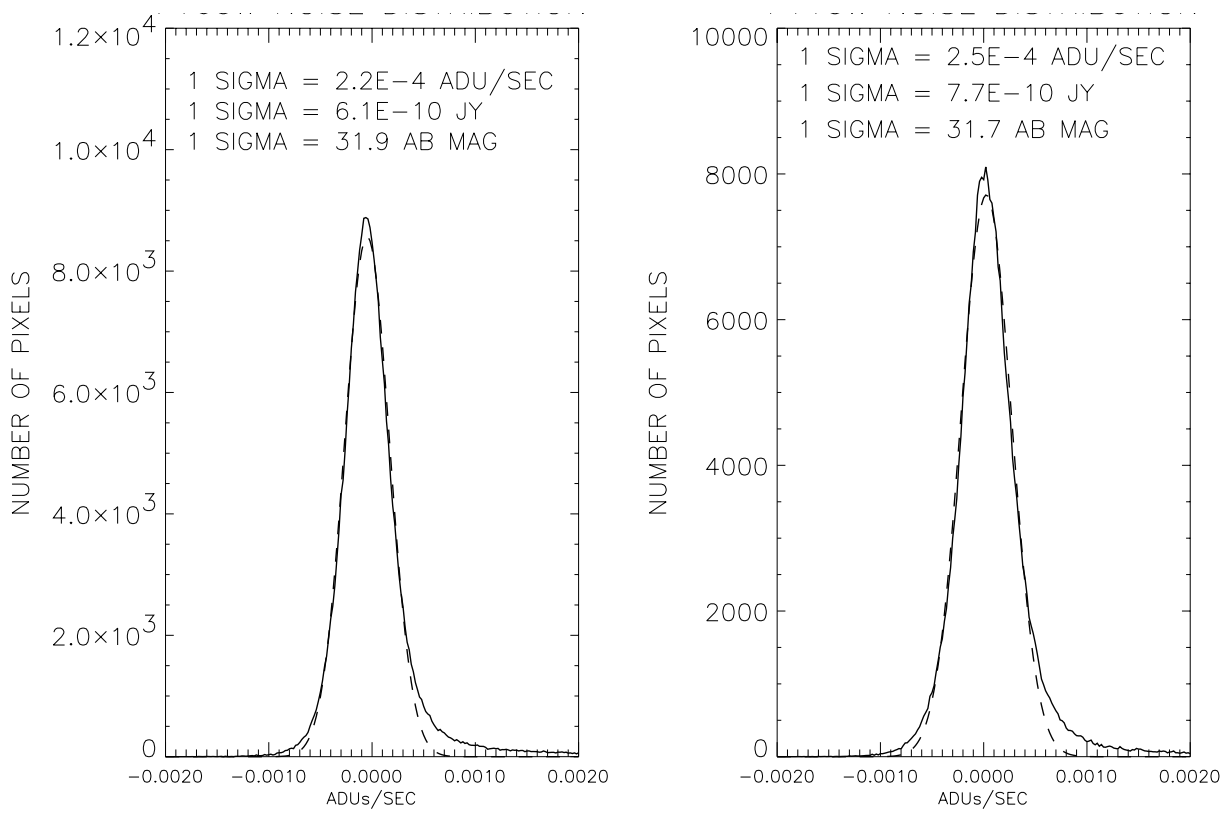

FIG. 5.-Histograms of the pixel values in the F160W (left) and F110W (right) mosaic frames are shown. Gaussian fits are overplotted as dotted lines. The measured $1 \sigma$ noise levels per pixel are $2.2 \times 10^{-4} \mathrm{ADUs} \mathrm{s}^{-1}\left(6.1 \times 10^{-10} \mathrm{Jy}, 31.9 \mathrm{AB}\right.$ mag $)$ for the F160W image and $2.5 \times 10^{-4} \mathrm{ADUs} \mathrm{s}^{-1}\left(7.7 \times 10^{-10} \mathrm{Jy}\right.$, $31.7 \mathrm{AB}$ mag) for the F110W image. The true noise levels are about a factor of 2 higher than these values owing to the correlated noise produced in the drizzling process.

smoothing the data is a $3 \times 3$ matrix that mimics the PSF of the central star in the drizzled data. This is much more sharply peaked than the Gaussian function used in the SExtractor analysis.

\subsubsection{Preparation of the Detectivity Image}

Each pixel, pix $x_{i, j}$, in the final image has a quality $Q_{i, j}$ value associated with it. The quality value is the square root of the sum of the squares of the total efficiency of each pixel in the individual image that contributes to the final image. Owing to the raster and dither pattern, a pixel in the final image has contributions from many different individual image pixels.

$$
Q_{i, j}=\sqrt{\sum_{k=1}^{n}\left(\mathrm{eff}_{k}\right)^{2}} .
$$

Here $n$ is the number of pixels contributing to the final image pixel, $\mathrm{pix}_{i, j}$, and the efficiency eff $_{k}$ of each contributing pixel is measured by the inverse of its multiplicative flat-field value. Figures 6 and 7 show the detectivity functions over the image areas used in the catalog.

\subsection{SExtractor Reduction}

As a cross-check on the KFOCAS detections we utilized an alternative source extraction system, SExtractor, on data

TABLE 2

KFOCAS PARAMETERS

\begin{tabular}{ccc}
\hline \hline KFOCAS Parameter & F160W & F110W \\
\hline Magnitude zero point $\ldots \ldots \ldots \ldots \ldots \ldots \ldots \ldots$ & 35.3 & 35.186 \\
Catalog magnitude limit $\ldots \ldots \ldots \ldots \ldots \ldots \ldots$ & 100. & 100. \\
Radius of fixed circular aperture $\ldots \ldots \ldots \ldots \ldots$ & 6.0 & 6.0 \\
Sigma of sky $\ldots \ldots \ldots \ldots \ldots \ldots \ldots \ldots \ldots \ldots \ldots$ & 20 & 22 \\
Sigma above sky for detection $\ldots \ldots \ldots \ldots \ldots \ldots$ & 2.5 & 3.0 \\
Sigma below sky for detection $\ldots \ldots \ldots \ldots \ldots \ldots$ & 20.0 & 20.0 \\
Minimum area for detection $\ldots \ldots \ldots \ldots \ldots \ldots \ldots$ & 2 & 2 \\
Significance for evaluation and splitting $\ldots \ldots \ldots$ & 0.15 & 0.15 \\
\hline
\end{tabular}

reduced via the IRAF reduction procedure rather than the IDL based procedure.

\subsubsection{Galaxy Detection}

We performed object detection on the F160W images and photometry on the F160W and F110W images using SExtractor version 2.0.7 (Bertin \& Arnouts 1996). The final F160W and F110W images reduced in the IRAF pipeline showed low-frequency structure in the background in the

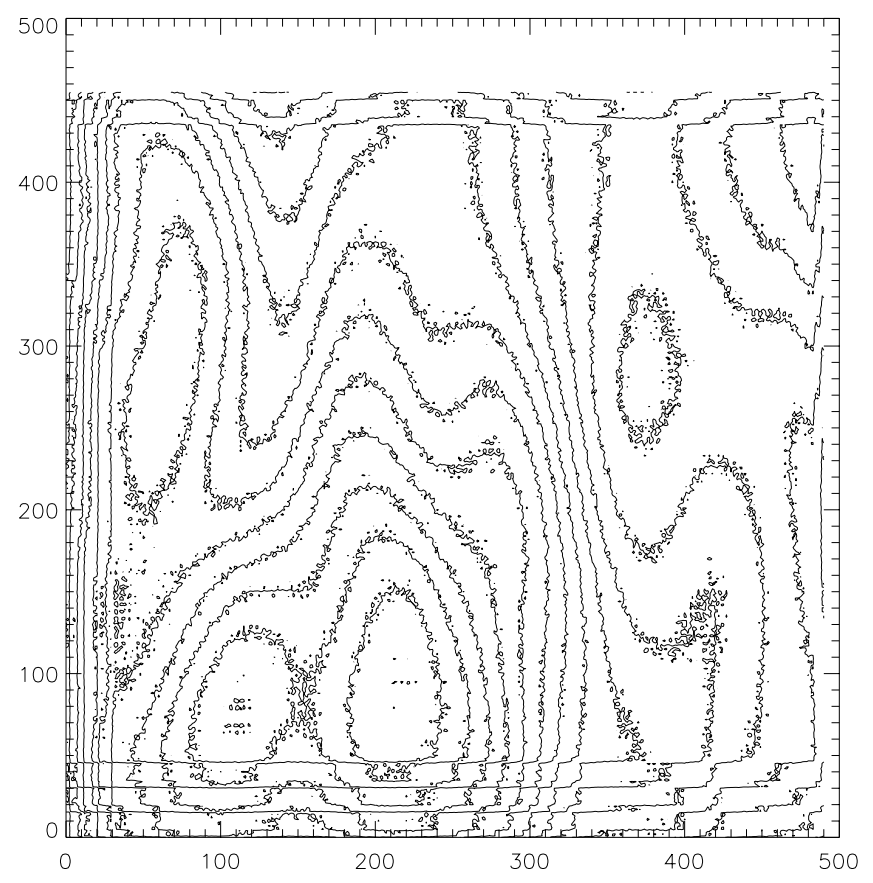

FIG. 6.-F160W source detectability contours for the region included in the catalog. The contours inside the F160W area cover a factor of 3.77. The contour levels are $5 \%$ of this range. The regions with the highest detectivity are in the center and lower left. 


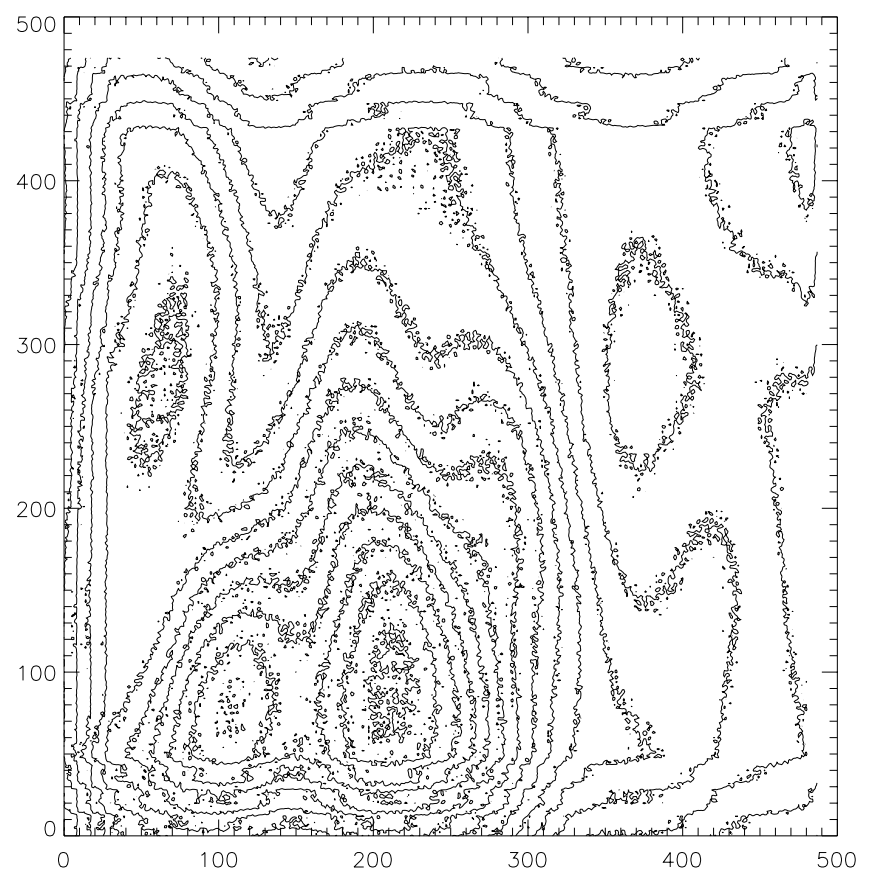

FIG. 7.-F110W source detectability contours for the region included in the catalog. The contours inside the F110W area cover a factor 4.75. The contour levels are $5 \%$ of this range. The regions with the highest detectivity are in the center and lower left.

$X$-direction. We created background frames in SExtractor using a $64 \times 32$ mesh, which were subtracted from the reduced frames, producing cosmetically more uniform backgrounds. We measure $1 \sigma$ noise levels from histograms of all the pixels in the frames of $2.0 \times 10^{-4} \mathrm{ADUs} \mathrm{s}^{-1}$ in the F160W image and $2.4 \times 10^{-4}$ ADUs s ${ }^{-1}$ in the F110W frame, consistent with the values shown in Figure 5. The amplitude of the fluctuations in the background varies by up to $50 \%$ across the image owing to variations in the quantum efficiency of the detector and the dither pattern used. Thus we used the option in SExtractor (WEIGHT_TYPE) that accepts a user-supplied variance map (for which we used the "detectivity" function described in $\S$ 7.2.1). SExtractor robustly scales the weight map to the appropriate absolute level by comparing the weight map to an internal, low-resolution, absolute variance map built from the science image itself. In contrast to the KFOCAS source extraction, all object detection was done on the F160W image, and magnitudes were measured to the corresponding isophotes on the F110W image.

After experimenting with different values of SExtractor parameters, we adopted the values given in Table 3. Aside from the determination of the local variance itself, the three most critical parameters that affect the detection of very faint isolated sources are FILTER_NAME, DETECT_MINAREA, and DETECT_THRESH. The FILTER_NAME parameter describes the smoothing kernel that is applied to the image, and for this a Gaussian with a full width half-maximum of 2.0 (drizzled) pixels was used over a $3 \times 3$ pixel grid. We tested various combinations of DETECT_MINAREA, the minimum number of contiguous pixels above a level that is the product of the parameter DETECT_THRESH times the local rms fluctuation in the background. Our final choices for these parameters are DETECT_MINAREA $=2$ pixels
TABLE 3

\begin{tabular}{|c|c|}
\hline Parameter & Value \\
\hline DETECT_MINAREA . & 2 \\
\hline DETECT_THRESH .......... & 2.15 \\
\hline ANALYSIS_THRESH ......... & 2.15 \\
\hline FILTER_NAME ............. & gauss_2.0_3 3 3.conv \\
\hline CLEAN $\ldots \ldots \ldots \ldots \ldots \ldots \ldots \ldots$ & $\mathrm{N}$ \\
\hline MASK_TYPE .............. & CORRECT \\
\hline WEIGHT_TYPE ............. & MAP_WEIGHT \\
\hline PHOT_APERTURES ........ & 6 \\
\hline 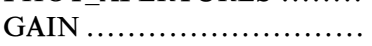 & 6.5 \\
\hline PIXEL_SCALE ............... & 0.1 \\
\hline SEEING_FWHM ............. & 0.2 \\
\hline BACK_SIZE ................. & 64 \\
\hline BACK_FILTERSIZE ......... & 3 \\
\hline BACKР̈НОTO_ТYPE ....... & LOCAL \\
\hline BACKPHOTO_THICK....... & 24 \\
\hline MAG_ZEROPOINT ........... & $22.80(\mathrm{~F} 160 \mathrm{~W})$ \\
\hline MAG ZEROPOINT ......... & 22.68 (F110W) \\
\hline
\end{tabular}

and DETECT_THRESH $=2.15 \sigma$. This choice for DETECT_MINAREA (the same value as for the equivalent KFOCAS parameter) favors slightly the detection of the most compact sources, and the final choice of 2.15 for DETECT_THRESH was dictated by an attempt to strike a judicious balance between completeness and reliability. We tested an alternative set of parameters with DETECT_MINAREA set to 3 pixels and with DETECT_THRESH to a lower value in order to detect the same number of sources as with DETECT $\_$MINAREA $=2$ and DETECT_THRESH $=2.15$. Estimating the number of false detection rates and completeness as discussed in $\$ \S 9$ and 10, we found that these two sets of parameters behaved very similarly. For the reason stated above, we selected DETECT_MINAREA $=2$. Sections 9 and 10 contain a more quantitative discussion of the completeness and reliability of the detected sources.

Although as with the KFOCAS reductions we have deliberately erred on the side of extracting a fairly large estimated fraction of false detections (at the faintest levels) and a completeness level that is only of order $50 \%$, the catalog listings contain only sources with signal-to-noise ratios greater than 2.5 .

\subsection{Comparison of the IDL-KFOCAS and the IRAF-SExtractor Photometry}

At this point our analysis contains four components, the IDL and IRAF reduced images and the KFOCAS and SExtractor source extractions and photometry. In the spirit of independent cross-checks, it is useful to compare these results and to see if any differences lie primarily in the images or in the source extraction procedures. We will discuss the differences in completeness and reliability between the two methods after $\S \S 9$ and 10 . Data presented so far have been for either the IDL-KFOCAS or IRAFSExtractor procedures. The third panel in Figure 8 shows a comparison between the aperture magnitudes found by KFOCAS in the IDL image and the aperture magnitudes found by SExtractor in the IRAF images for all objects detected in common. The delta magnitudes are KFOCAS magnitude minus SExtractor magnitude. In both cases the diameter of the aperture is 0.6 . 

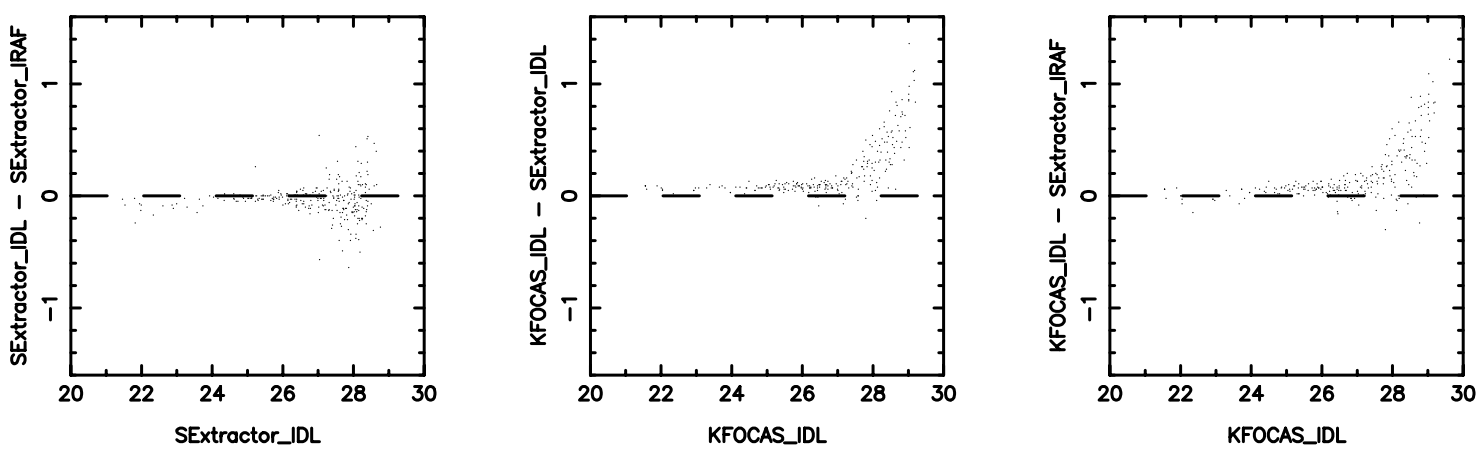

FIG. 8.-Comparison of KFOCAS and SExtractor aperture magnitudes before the catalog cut at $2.4 \sigma$. The first panel shows the difference in SExtractor magnitudes determined from the IDL and IRAF images. The second and third panels indicate the differences between the two source extraction programs, KFOCAS and SExtractor. The last panel is a good guide in comparing the SExtractor determined magnitudes in the catalog with those determined by KFOCAS

As expected, the correlation is very good at bright magnitudes and gets worse at the faint end. The SExtractor magnitudes are equal to the KFOCAS magnitudes up until 28th magnitude, where the SExtractor magnitudes become significantly brighter than the KFOCAS magnitudes. These differences could be due to differences either in the input images or in the magnitudes extracted by KFOCAS and SExtractor. To check this, we ran SExtractor on both the IDL-reduced and the IRAF-reduced images. The first panel in Figure 8 shows the result of this test, which shows a uniform scatter about the zero level at the fainter magnitudes. The slight offset at the bright end is probably due to the IRAF procedure IMCOMBINE clipping some pixels in the brightest galaxies in the IRAF image. A follow-up test comparing the KFOCAS reductions of the IDL image with SExtractor reductions of the IDL images is shown in the middle panel of Figure 8. This plot is essentially identical to the last panel except for about a 0.07 mag offset at the brighter end. This set of tests shows that the differences between the KFOCAS/IDL magnitudes and the SExtractor/IRAF magnitudes shown in the last panel of Figure 8 are entirely due to the differences between the KFOCAS and SExtractor algorithms, not from any differences between the IDL and IRAF image production procedures. The origin of this difference is not clear, but the reader should be aware that these two standard procedures do produce differences at the very faintest levels.

\section{THE CATALOG}

Table 4 contains the catalog of sources from the KFOCAS source extraction from the F160W and F110W images. This catalog contains only sources with signal-tonoise ratios greater than 2.5 . We anticipate a future publication describing the sources with less reliable detections. The catalog contains 342 objects, some of which are components of a larger object. The catalog contains 235 objects in common with the WFPC2 catalog. Two-hundred twenty-one objects have detections in both filters, 56 objects have a detection only in the F160W filter, 53 have a detection only in the F110W filter, and none have detections only in SExtractor. The objects are arranged by right ascension, which sometimes separates different components of the same object in the catalog. The data and numbering in the catalog have the priorities, in order, of F160W KFOCAS, F110W KFOCAS, and SExtractor. This means that all
KFOCAS objects detected in both filters use the KFOCAS F160W R.A., decl., and $x$ and $y$ positions. The magnitudes come from the KFOCAS F160W and F110W extractions. Positional coincidence is relative to the F160W positions. Objects that have F110W KFOCAS detections but not KFOCAS F160W detections use the F110W KFOCAS positions and magnitudes. The catalog columns contain the following parameters.

ID.-This is a running number for each object. The numbers after the decimal point indicate the level of splitting by KFOCAS up to three levels of daughter objects. Since the list is arranged by right ascension, daughter objects can appear separately from the parent objects. No object is repeated. Numbers of 900 or higher are split F110W objects that are not coincident with any F160W split even though some of the components are in common.

WFPC.-The WFPC column lists the nearest WFPC2 source from the Williams et al. (1996) catalog. This is not necessarily the same object, just the nearest.

$s$. - The $s$-value is the separation in arcseconds between the NICMOS and the nearest WFPC2 object as listed in the WFPC column. A large value of separation indicates that the NICMOS and WFPC2 object are probably not associated.

$x$ and $y$.-These columns give the $x$ - and $y$-values of the centroid of the source in the F160W or F110W image. If the object is detected in both images, the $x$ - and $y$-values refer to the F160W image. Objects detected only by SExtractor have the values determined by SExtractor. This order of precedence holds for all of the subsequent values.

R.A. and Decl.-These columns give the right ascension and declination of the centroid of the source. Only the minutes and seconds are listed. The hour of right ascension is $12^{\mathrm{h}}$ for all sources, and all sources have $62^{\circ}$ of declination. The source positions assume that the central star, NICMOS 145, and the WFPC2 object 4-454 have the same position and that the measured plate scales of the NICMOS Camera 3 are correct. In this sense all positions are relative to the position of the WFPC2 4-454 object.

$t_{160}, i_{160}, a_{160}, t_{110}, i_{110}$, and $a_{110}$. - These are the total, isophotal, and aperture magnitudes found by KFOCAS in the F160W and F110W images. The aperture diameter for the aperture magnitudes is 0.6 . The total and isophotal magnitudes are as described in Williams et al. (1996). A value of 99.99 indicates that the object was not detected in 


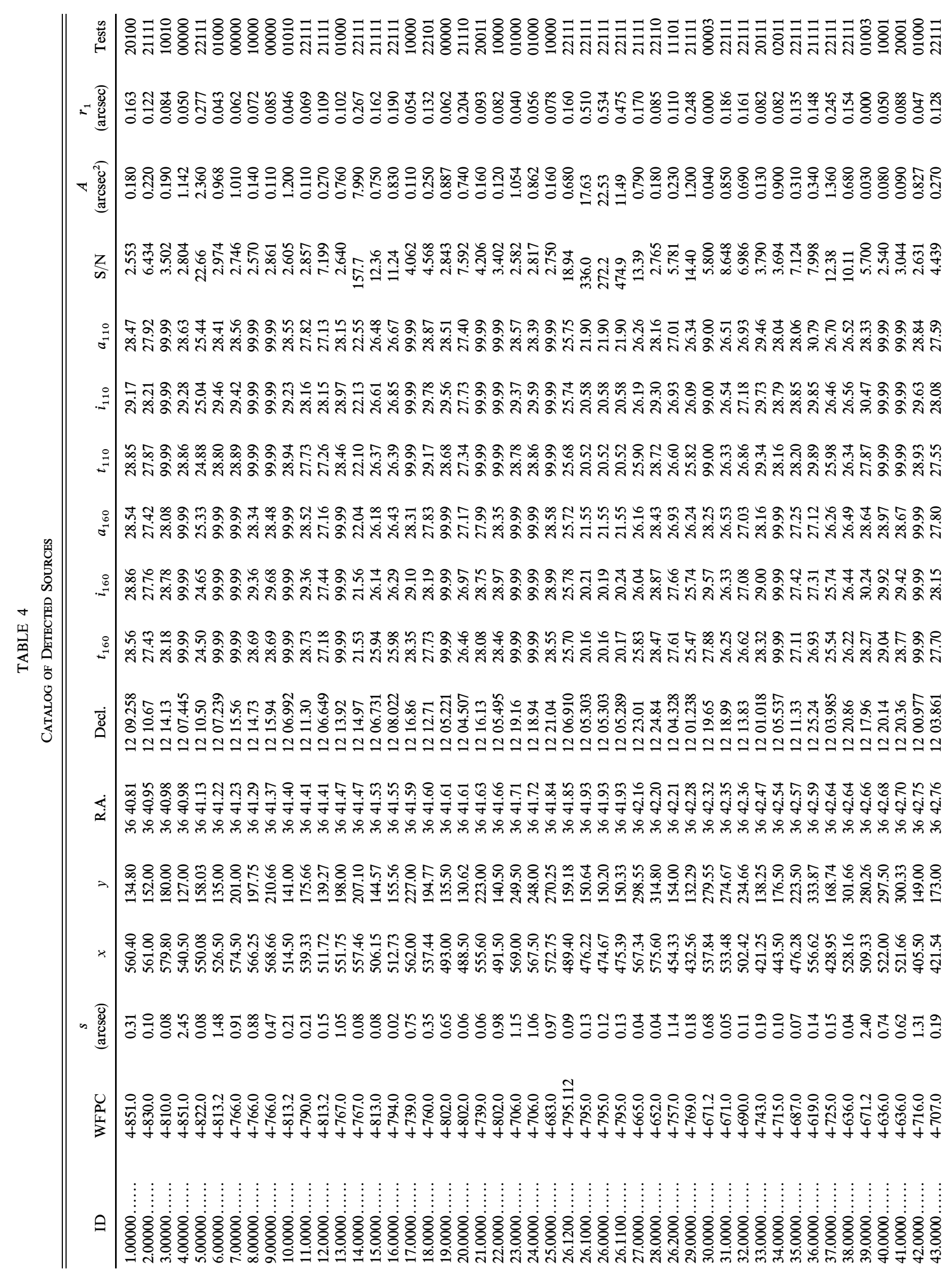




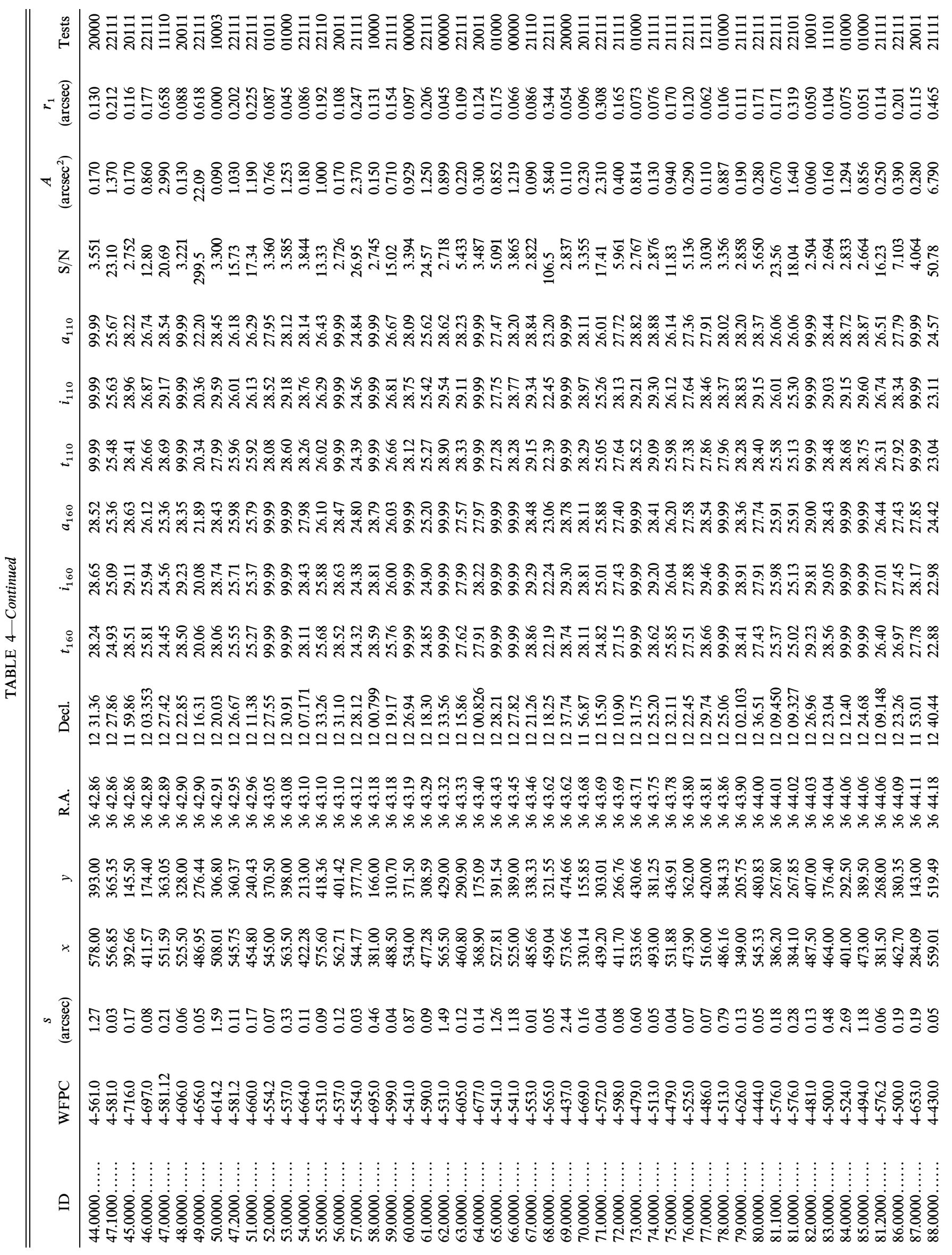




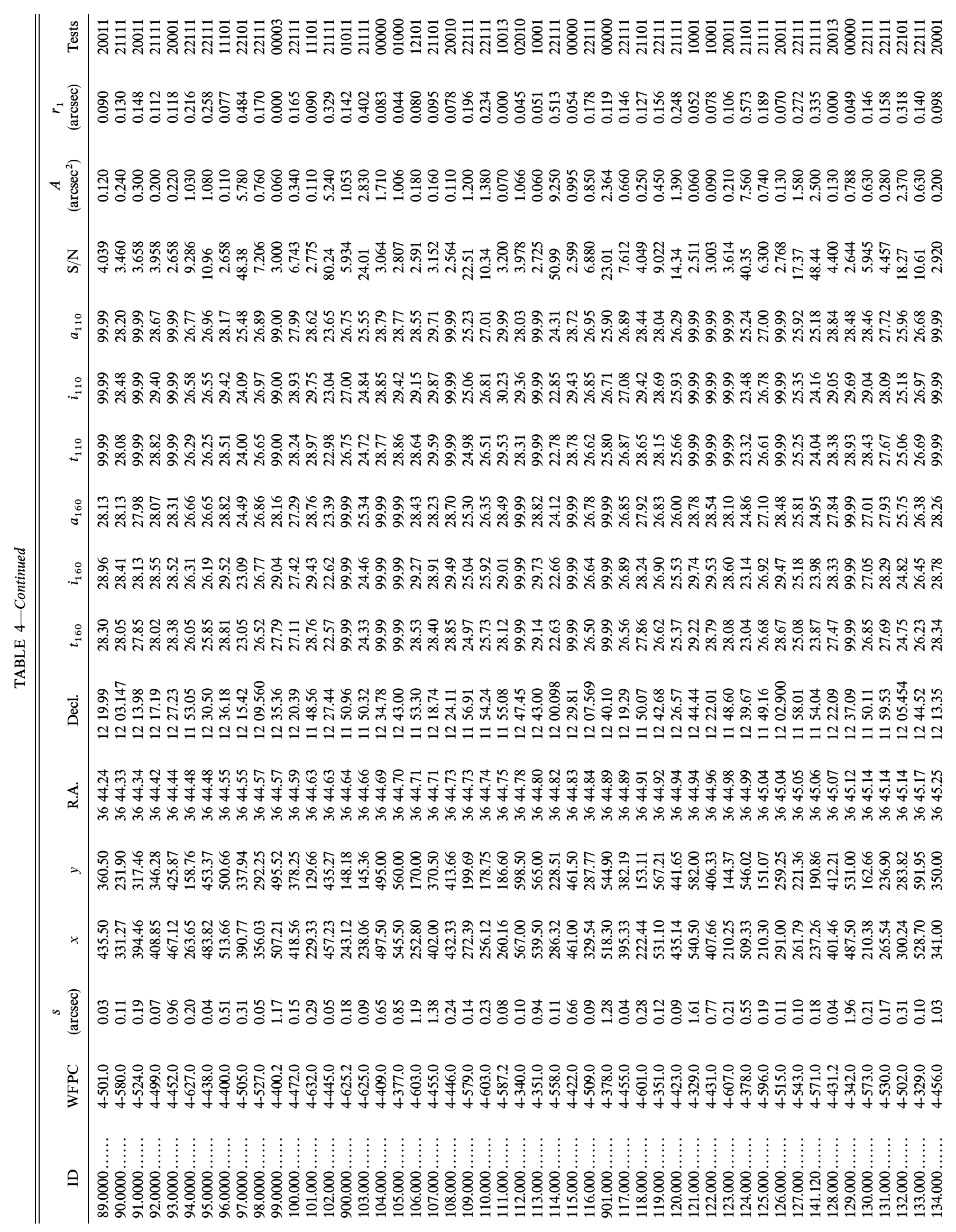









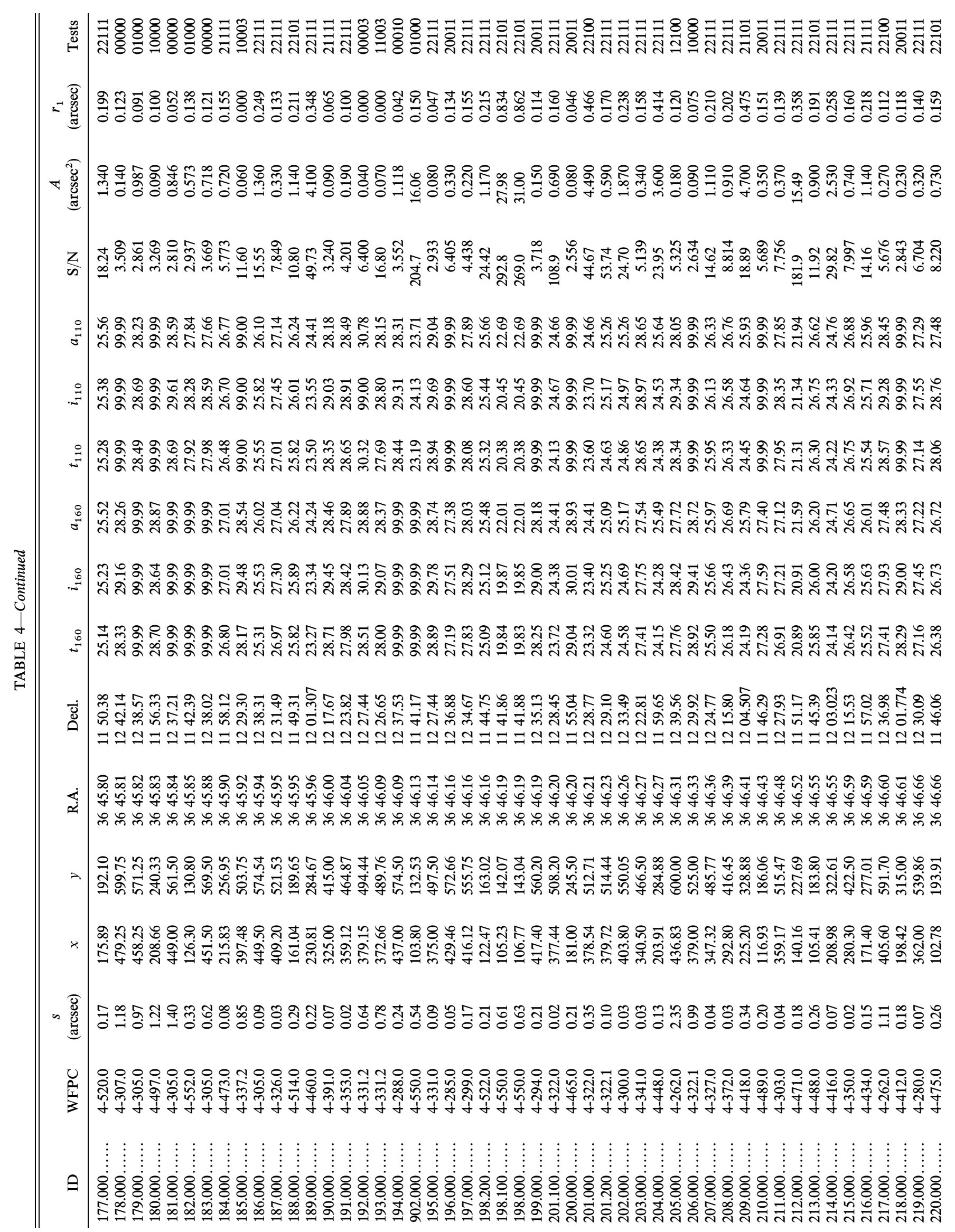




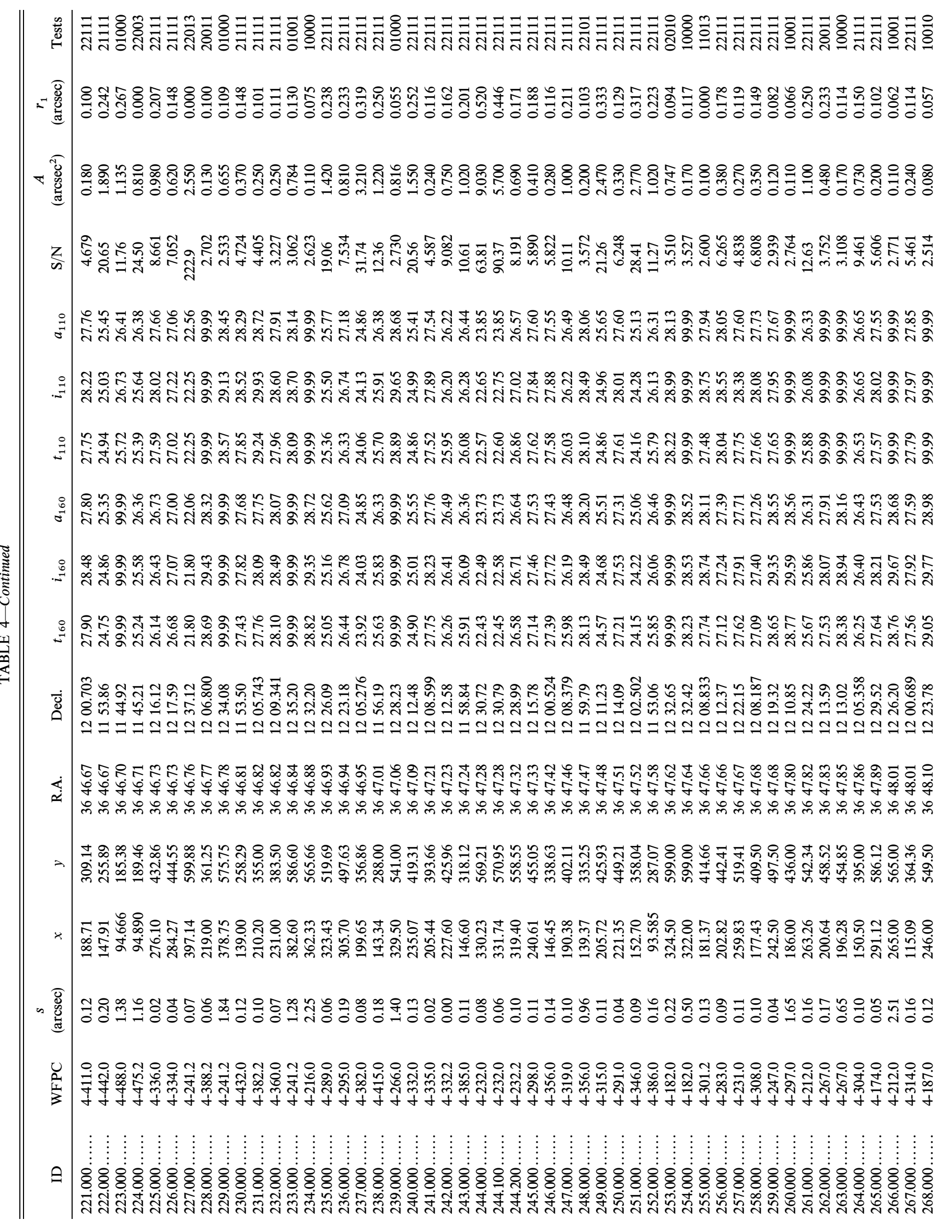




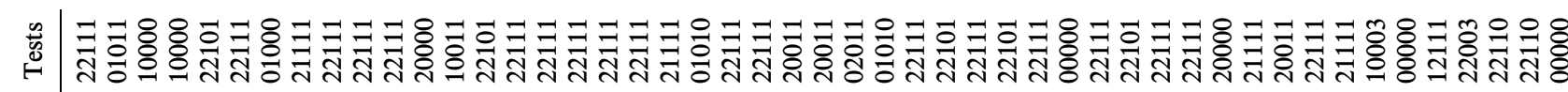

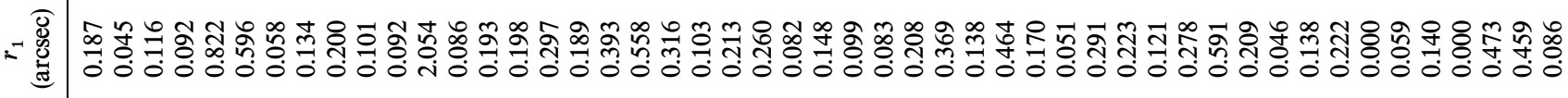

『苞

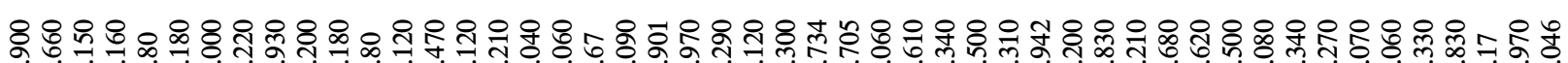

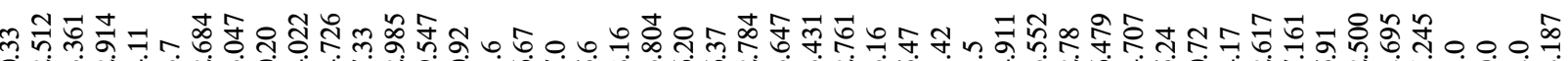

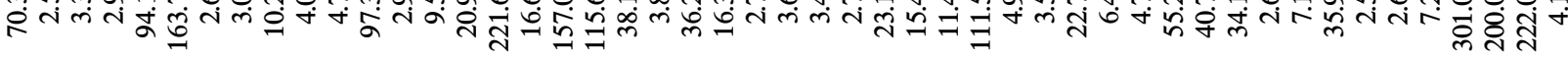

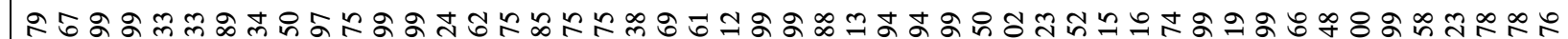

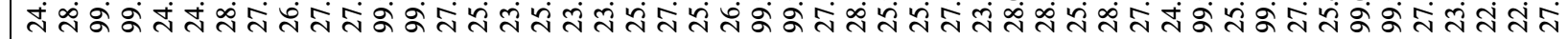

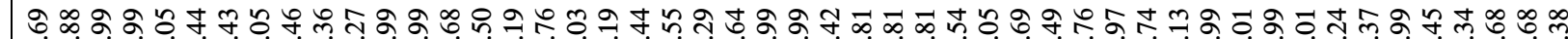

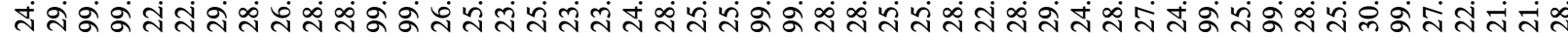

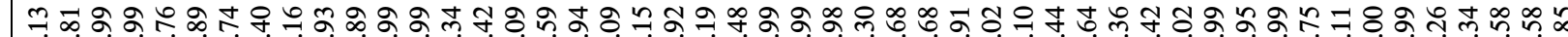

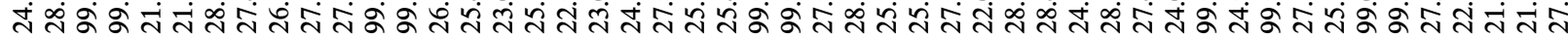

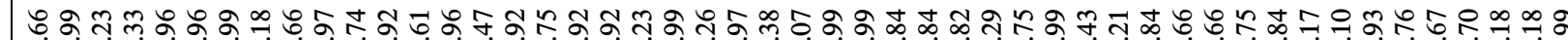

\section{$=$}

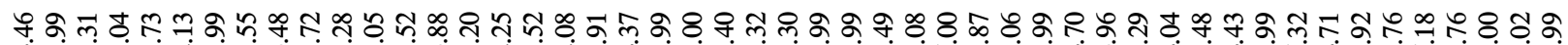

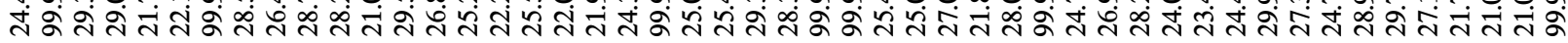

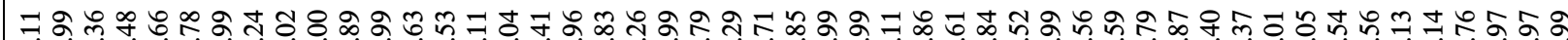

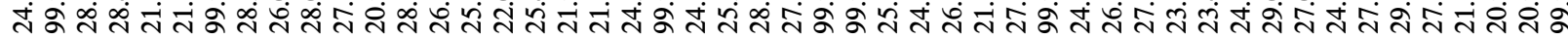

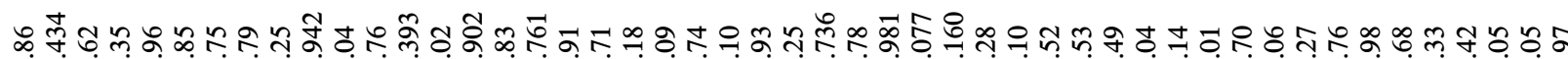

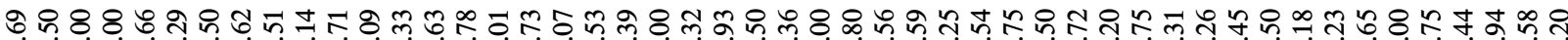

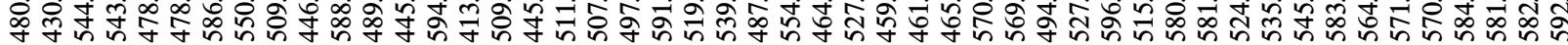

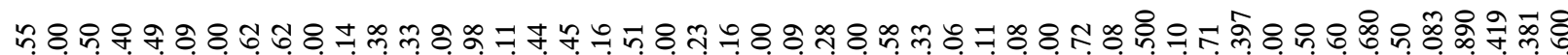

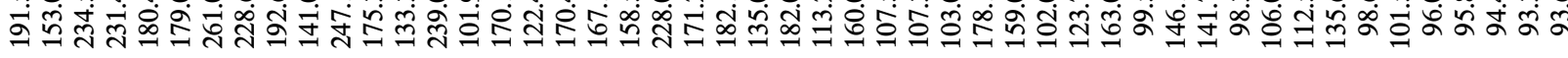

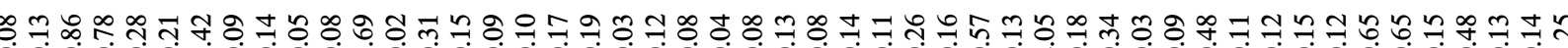

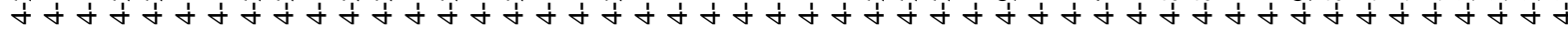

А

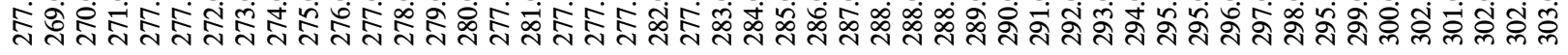


that filter. The F160W and F110W objects are considered to be in common if they lie within 0 "'.25 of each other. If the last digit in the tests parameter (see below) is 3, the magnitudes are from the SExtractor procedure.

$S / N$.-The signal-to-noise ratio value quoted in the catalog is calculated by the same technique used in the optical HDF. The value of the signal-to-noise is $\mathrm{S} / \mathrm{N}=$ $L_{i} / \sigma\left(L_{i}\right)$, where the variance $\left[\Gamma \sigma\left(L_{i}\right)\right]^{2}$ is

$\left[\Gamma \sigma\left(L_{i}\right)\right]^{2}=\Gamma N_{\mathrm{obj}}+1.9^{2} \Gamma^{2} \sigma_{\mathrm{sky}}^{2} A_{\mathrm{obj}}+1.9^{2} \Gamma^{2} \sigma_{\mathrm{sky}}^{2} A_{\mathrm{obj}}^{2} / A_{\mathrm{sky}}$

as quoted in its correct form by Pozzetti et al. (1998). For NICMOS, the value of $\Gamma$ in electrons per ADU is 6.5. $L_{i}$ is the sky-subtracted number of counts, $\sigma_{\text {sky }}$ is the sky sigma in ADUs, and the object and sky areas $A_{\text {sky }}$ and $A_{\text {obj }}$ are the areas in pixels returned by KFOCAS. This equation reformulated in terms of count rates in ADUs s ${ }^{-1}$ is given by

$$
\sigma_{\text {tot }}^{2}=\operatorname{rate}_{\text {obj }} /(\Gamma t)+1.9^{2} \sigma_{\text {skyrate }}^{2} A_{\text {obj }}+1.9^{2} \sigma_{\text {skyrate }}^{2} A_{\text {obj }}^{2} / A_{\text {sky }} \text {. }
$$

Here rate $_{\mathrm{obj}}$ is the sky-subtracted source count rate in ADUs s ${ }^{-1}, \sigma_{\text {skyrate }}$ is the sky sigma value in $\mathrm{ADUs} \mathrm{s}^{-1}$, and $t$ is the integration time in seconds. The final signal-to-noise ratio is rate ${ }_{\text {obj }} / \sigma_{\text {tot }}$. The measured value of the sky sigma is $2.2 \times 10^{-4} \mathrm{ADUs} \mathrm{s}^{-1}$ for the F160W image and $2.5 \times 10^{-4}$ for F110W as shown in Figure 2. The factor of 1.9 in each of the equations is the estimated value of the noise correlation discussed earlier. Figure 9 shows a plot of the signal-tonoise ratios calculated by this method for the KFOCAS determined sources.

A.-This is the isophotal area of the source in square arcseconds as determined from the value returned by KFOCAS.

$r_{1}$.-This is the half-light radius returned by KFOCAS.

Tests.-This parameter indicates which of the various reliability tests the source passed. A source that passed all tests has a value of 22111 , one that passed no tests has a value of 00000 . The first number is 0,1 , or 2 if the source was detected in none, one, or both of the F160W halfcatalogs. For an explanation of the half-catalogs see $\S 10.1$. The second number is the same test in the F110W catalog. The third number is 0 or 1 depending on whether the source was found in both NICMOS bands of the full image extrac-

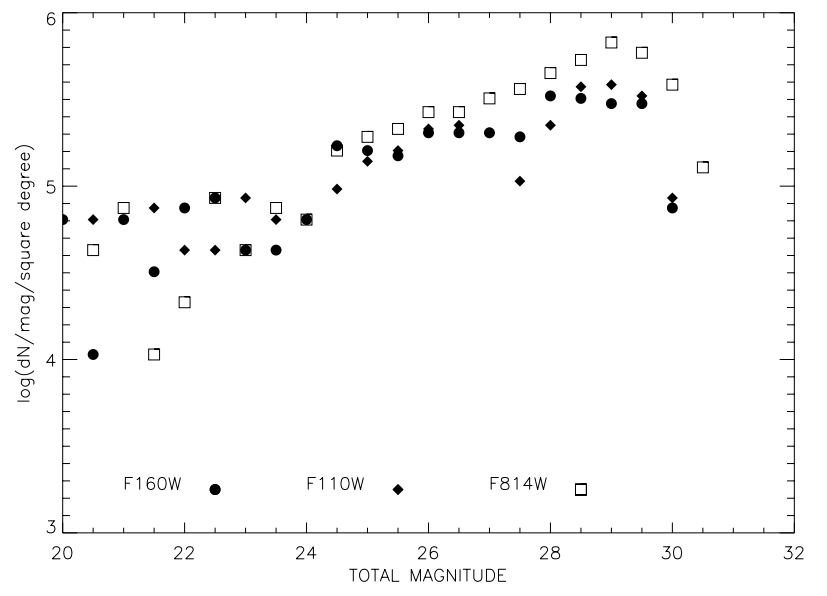

FIG. 9.-Signal-to-noise ratios for faint sources relative to the KFOCAS aperture magnitudes for the F160W and F110W filters. This figure contains all of the extracted sources, not just the catalog sources. tions. The fourth number is 0 or 1 depending on whether the source is detected in the WFPC2 catalog. The last number is 0 or 1 depending on whether the source was found in the independent Sextractor catalog. Note the discussions in $\$ \S 10.2$ and 10.3 on the differences in half-catalog detection probabilities between KFOCAS and SExtractor. In all cases a common detection means that the source centroids lie within 0.25 of each other. No color or magnitude tests are applied as part of the common object association.

\section{COMPLETENESS}

Our calculated $50 \%$ completeness levels are KFOCAS $\mathrm{AB}$ aperture magnitudes of 28.7 and 28.2 for point objects and extended sources in the F160W filter and KFOCAS AB magnitudes of 28.6 and 28.0, respectively, in the F110W filter. These limits are based on a technique of adding sources to the image at various flux levels and running the source extraction programs to see what percentage of the added sources are recovered. These numbers are based on the KFOCAS reductions.

\subsection{KFOCAS Completeness}

The test for the KFOCAS program established a regular grid of 49 positions in a $7 \times 7$ pattern that evenly covered the image area utilized in the catalog extraction. Sources are placed at these positions to create a new image that contains the original image plus the added sources. KFOCAS then creates a new catalog utilizing exactly the same parameters used in the final catalog preparation. An automatic program checks the new catalog to see what percentage of the added sources are recovered by KFOCAS. The sources are sequentially dimmed in half-magnitude steps from their original magnitudes of about 21 to the final magnitude of 32. The added sources are real sources extracted from the final image. The NICMOS source 145, the central star, represents a point object and the elliptical galaxy, NICMOS 212 , is the extended object. These are WFPC2 sources 4-454 and 4-471, respectively. Alteration of the position of the grid confirmed that the completeness limits were consistent at different grid positions. The extended source completeness limit will of course be brighter for more extended objects than NICMOS 212, but this galaxy is one of the largest in the image.

The completeness value at a given magnitude is for the magnitude of the input source, not the magnitude at which the source is recovered. This value is approximately equal to the recovered magnitude for sources brighter than 26.5. At the very faintest levels, the recovered magnitudes are $0.3-0.5 \mathrm{mag}$ fainter than the input magnitudes. The measured completeness limits are fitted by

$$
\left(1-\frac{m-m_{b}}{m_{a}-m_{b}}\right)^{n} \sqrt{\frac{m_{b}}{m}} .
$$

In this function $m_{a}$ is the magnitude at which the completeness goes to zero, $m_{b}$ is the magnitude at which the completeness is $100 \%$, and the power index $n$ is adjusted to fit the data. The function is purely empirical, simply designed to fit the data well. This function smooths the curves and provides the interpolation from the observed magnitudes to the magnitudes used in Table 5. As expected from the nonuniform quality map of the image, the completeness limit is not uniform over the image. The complete- 
ness is somewhat lower in the right-hand half of the image. The completeness limits in Table 5 should be considered as an average across the image.

\subsection{SExtractor Completeness}

For the SExtractor catalog we used an analysis similar to the one described in Yan et al. (1998). We selected a compact galaxy (4-289 in the Williams catalog), representative of the majority of the objects in our field, and dimmed it in half-magnitude increments from 25.5 to 29. The dimmed galaxy was dropped at random positions 10,000 times, superposed on the full image and two images with half the exposure time of the full image. (The "half-" images are discussed in $\S 10.1$.) We ran SExtractor at the position at which the galaxy was dropped at each iteration to determine whether or not the dimmed galaxy was recovered and at what magnitude. The use of random positions in the simulations allows us to include completeness corrections arising from nondetections and magnitude errors caused by crowding and spatially dependent errors in the sky subtraction and flat-field correction. As discussed in $\S 9.1$, the completeness values for recovery of the images are again an area average of the completeness since the sensitivity across the NICMOS images is not uniform. These same experiments also give us the matrix relating the input and output aperture magnitudes for those galaxies that are recovered. The input magnitude and mean recovered magnitude agree to within 0.07 mag in the bins through 28. At 28.5 the mean of the recovered galaxy magnitudes begins to brighten (28.21), and by an input magnitude of 29 it brightens substantially (28.11). The galaxies that land on negative noise regions are lost completely. The SExtractor catalog becomes $50 \%$ complete at $\mathrm{AB} \approx 28.3$ for the compact galaxies in our survey.

To test differences in detectability of the point-spread function due to the source landing at different locations within the 0.2 pixels, we ran incompleteness tests using the central star (NICMOS $145, \mathrm{AB}=22.1$ ) taken from five different individual exposures, all taken at different dither positions. These have only $1 / 120$ the exposure time of the final image and therefore have lower signal-to-noise ratios. We found no substantial differences in detection rates, other than as the star is dimmed to $\mathrm{AB}=28$, it is missed most frequently in the upper right-hand quadrant of the detector, the least sensitive region of the image. In the final combined image, we found no obvious location dependence. We detect the star $95 \%$ of the time as it is dimmed to $\mathrm{AB}=27$, and then the detection rate drops rapidly to $50 \%$ complete at $\mathrm{AB}=28.5$.

\section{SOURCE RELIABILITY TESTS}

Even though the listed catalog does not contain objects with signal-to-noise ratios less than 2.5 , there can be false detection still in the catalog. As indicated in $\S 8$, the catalog indicates the degree of coincidence between the various subcatalogs that make up the total catalog. These data are provided as an aid in discerning the reality of the sources. Any statistical study of these results should utilize the test flag indices of Table 4 carefully along with the completeness and reliability results discussed here and in $\S 9$ and summarized in Table 5. See $\S 10.4$ for a discussion of this table. Table 6 presents a catalog of detected sources with signalto-noise ratios less than 2.5 .

TABLE 5

COMPLETENESS AND RELIABILITY

\begin{tabular}{|c|c|c|c|c|c|c|c|c|c|c|}
\hline \multirow[b]{2}{*}{$\begin{array}{l}\text { MAGNITUDE }^{\mathrm{a}} \\
\text { (1) }\end{array}$} & \multirow[b]{2}{*}{$\begin{array}{c}\mathrm{S} / \mathrm{N} \\
(2)\end{array}$} & \multicolumn{5}{|c|}{ KFOCAS } & \multirow[b]{2}{*}{$\begin{array}{c}R_{11} \\
(8)\end{array}$} & \multicolumn{3}{|c|}{ SX } \\
\hline & & $\begin{array}{c}C s_{16} \\
(3)\end{array}$ & $\begin{array}{c}C l_{16} \\
\text { (4) }\end{array}$ & $\begin{array}{c}C s_{11} \\
(5)\end{array}$ & $\begin{array}{c}C l_{11} \\
\text { (6) }\end{array}$ & $\begin{array}{c}R_{16} \\
\text { (7) }\end{array}$ & & $\begin{array}{c}C_{16} \\
(9)\end{array}$ & $\begin{array}{c}R_{\text {co } 16}{ }^{\mathrm{b}} \\
\quad(10)\end{array}$ & $\begin{array}{l}R_{\text {neg } 16^{\mathrm{c}}} \\
\quad \text { (11) }\end{array}$ \\
\hline $20.000 \ldots \ldots$ & 283.8 & 100.0 & 100.0 & 100.0 & 100.0 & 1.000 & 1.000 & $\ldots$ & $\ldots$ & $\ldots$ \\
\hline $20.500 \ldots \ldots$ & 287.9 & 100.0 & 100.0 & 100.0 & 100.0 & 1.000 & 0.833 & $\ldots$ & $\ldots$ & $\ldots$ \\
\hline $21.000 \ldots \ldots$ & 166.5 & 100.0 & 100.0 & 100.0 & 100.0 & 1.000 & 0.750 & $\ldots$ & $\ldots$ & $\ldots$ \\
\hline $21.500 \ldots \ldots$ & 195.8 & 100.0 & 100.0 & 100.0 & 100.0 & 1.000 & 0.750 & $\ldots$ & $\ldots$ & $\ldots$ \\
\hline $22.000 \ldots \ldots$. & 114.2 & 100.0 & 98.85 & 100.0 & 100.0 & 1.000 & 0.800 & $\ldots$ & $\ldots$ & $\ldots$ \\
\hline $22.500 \ldots \ldots$ & 125.0 & 100.0 & 97.75 & 100.0 & 98.88 & 1.000 & 0.900 & $\ldots$ & $\ldots$ & $\ldots$ \\
\hline $23.000 \ldots \ldots$ & 79.12 & 100.0 & 96.67 & 100.0 & 97.79 & 1.000 & 0.875 & $\ldots$ & $\ldots$ & $\ldots$ \\
\hline $23.500 \ldots \ldots$ & 48.68 & 98.93 & 95.62 & 98.93 & 96.71 & 1.000 & 0.542 & $\ldots$ & $\ldots$ & $\ldots$ \\
\hline $24.000 \ldots \ldots$ & 58.21 & 97.89 & 94.53 & 97.89 & 95.58 & 1.000 & 0.916 & $\ldots$ & $\ldots$ & $\ldots$ \\
\hline $24.500 \ldots \ldots$ & 28.31 & 96.87 & 93.35 & 96.87 & 94.28 & 1.000 & 0.663 & $\ldots$ & $\ldots$ & $\ldots$ \\
\hline $25.000 \ldots \ldots$ & 22.32 & 95.83 & 91.91 & 95.83 & 92.63 & 0.961 & 0.732 & $\ldots$ & $\ldots$ & $\ldots$ \\
\hline $25.500 \ldots \ldots$ & 17.82 & 94.66 & 90.01 & 94.66 & 90.36 & 1.000 & 0.783 & 97.6 & $\ldots$ & $\ldots$ \\
\hline $26.000 \ldots \ldots$ & 12.59 & 93.14 & 87.31 & 93.14 & 87.11 & 0.973 & 0.806 & 96.2 & $\ldots$ & $\ldots$ \\
\hline $26.500 \ldots \ldots$ & 9.327 & 90.89 & 83.32 & 90.89 & 82.42 & 0.957 & 0.722 & 95.1 & $\ldots$ & $\ldots$ \\
\hline $27.000 \ldots \ldots$ & 7.192 & 87.28 & 77.38 & 87.28 & 75.68 & 0.977 & 0.601 & 92.2 & $\ldots$ & $\ldots$ \\
\hline $27.500 \ldots \ldots$ & 5.989 & 81.38 & 68.62 & 81.38 & 66.17 & 0.848 & 0.610 & 89.1 & $\ldots$ & $\ldots$ \\
\hline $28.000 \ldots \ldots$ & 4.852 & 71.85 & 55.91 & 71.85 & 53.01 & 0.681 & 0.458 & 76.6 & 0.94 & 0.87 \\
\hline $28.500 \ldots \ldots$ & 3.580 & 56.86 & 37.82 & 56.86 & 35.17 & 0.461 & 0.205 & 41.5 & 0.68 & 0.50 \\
\hline $29.000 \ldots \ldots$. & 2.889 & 33.96 & 12.57 & 33.96 & 11.44 & 0.247 & 0.122 & 14.1 & $\ldots$ & $\ldots$ \\
\hline $29.500 \ldots \ldots$ & 2.260 & 0.000 & 0.000 & 0.000 & 0.000 & 0.157 & 0.075 & $\ldots$ & $\ldots$ & $\ldots$ \\
\hline $30.000 \ldots \ldots$ & 1.635 & 0.000 & 0.000 & 0.000 & 0.000 & 0.123 & 0.079 & $\ldots$ & $\ldots$ & $\ldots$ \\
\hline
\end{tabular}

NoTE.-The brighter magnitude bins in the SExtractor columns have no measurements as no tests were run at these values. The completeness and reliability are assumed to be very high for $\mathrm{AB}<26.5$ as shown in the KFOCAS tests.

${ }^{a}$ For the KFOCAS columns the values refer to the magnitudes measured by KFOCAS. For the SExtractor columns the values refer to the magnitudes measured by SExtractor.

${ }^{\mathrm{b}} R_{\mathrm{co} 16}$ refers to the full coincidence formalism described in $\S 10.1 .1$.

${ }^{\mathrm{c}} R_{\text {neg } 16}$ refers to the negative image technique described in $\S 10.1 .2$. 
TABLE 6

Catalog of Detected Sources with Signal-to-Noise Ratio Levels Less than 2.5

\begin{tabular}{|c|c|c|c|c|c|c|c|c|c|c|c|c|c|c|c|c|}
\hline ID $^{a}$ & WFPC & $\begin{array}{c}s \\
(\operatorname{arcsec})\end{array}$ & $x$ & $y$ & R.A. & Decl. & $t_{160}$ & $i_{160}$ & $a_{160}$ & $t_{110}$ & $i_{110}$ & $a_{110}$ & $\mathrm{~S} / \mathrm{N}$ & $\begin{array}{c}A \\
\left(\operatorname{arcsec}^{2}\right)\end{array}$ & $\begin{array}{c}r_{1} \\
(\operatorname{arcsec})\end{array}$ & Tests \\
\hline 501.000 & 851.0 & 0.53 & 57.50 & 139.00 & 40.88 & 1209.423 & 29.98 & 29.85 & 30.34 & 99.99 & 99.99 & 99.99 & 1.255 & 0.060 & 0.051 & 20000 \\
\hline $502.000 \ldots$ & $4-807.0$ & 0.15 & 561.00 & 170.50 & 3641.11 & 1212.19 & 29.35 & 30.28 & 29.02 & 28.28 & 28.79 & 28.19 & 1.917 & 0.080 & 0.050 & 20111 \\
\hline $503.000 \ldots \ldots$ & $4-822.0$ & 1.01 & 543.50 & 151.00 & 3641.15 & 1209.547 & 29.99 & 29.89 & 29.69 & 99.99 & 99.99 & 99.99 & 1.240 & 0.060 & 0.051 & 10000 \\
\hline $504.000 \ldots \ldots$ & $4-822.2$ & 1.47 & 538.16 & 149.94 & 3641.19 & 1209.121 & 27.82 & 30.29 & 28.19 & 99.00 & 99.00 & 99.00 & 0.900 & 0.020 & 0.000 & 00003 \\
\hline $505.000 \ldots$ & $4-807.0$ & 1.71 & 559.33 & 188.16 & 3641.28 & 1213.54 & 28.64 & 28.82 & 28.64 & 99.99 & 99.99 & 99.99 & 2.365 & 0.180 & 0.119 & 20000 \\
\hline $506.000 \ldots \ldots$ & $4-766.0$ & 1.00 & 562.50 & 197.00 & 3641.33 & 1214.49 & 99.99 & 99.99 & 99.99 & 29.14 & 30.00 & 29.14 & 2.146 & 0.592 & 0.050 & 01000 \\
\hline
\end{tabular}

NoTE.-Table 6 is presented in its entirety in the electronic edition of the Astronomical Journal. A portion is shown here for guidance regarding its form and content. Units of right ascension are minutes and seconds, and units of declination are arcminutes and arcseconds.

${ }^{\text {a }}$ Sources with signal-to-noise ratio levels less than 2.5 have ID numbers of 500 or greater.

\subsection{Half-Data Reductions}

Our primary test of the reliability of observed sources utilizes two independent images formed from subsets of the integrations in each filter. The two images contain the evenand odd-numbered integrations from a sequential numbering of the integrations after removal of images with excess cosmic-ray persistence. Since there are three images per orbit, this technique ensures that orbits are mixed between the groups and that each group has an equal mix of images observed at different times during the orbit. The widths of the histograms of pixel values in the half-images are a factor of $\sqrt{2}$ wider than the full image histograms. This is a good indication that the width of the histogram in Figure 5 is due to noise rather than faint sources.

These half-data reductions are the primary tests as they represent truly independent sets of data that measure the same quantity. Although useful, the coincidences between the KFOCAS and SExtractor catalogs are not measurements of two independent data sets. The coincidences between the objects detected in the various NICMOS and WFPC2 filter sets are again useful, but they are not measuring the same quantity. As with the completeness tests, the checks on the KFOCAS and Sextractor image catalogs are carried out independently. With slight modifications, however, the logic of the tests is essentially identical.

\subsubsection{Logic of the Half-Catalog Tests}

Our goal is a measurement of the probability that a detected source with a given magnitude range is real. To facilitate the comparison between KFOCAS and SExtractor tests, we utilized 0"6 diameter aperture magnitudes in both source extraction programs. We start by grouping sources into half-magnitude bins centered on integer and half-magnitudes. Our analysis method then utilizes the statistics of objects detected in both, only one, or neither of the independent half-catalogs for each aperture magnitude bin. We consider objects as being present in both catalogs if their centroids are within 0.25 (2.5 drizzled pixels) of each other.

From the completeness studies described in $\S 9$ we determined the probability $P_{\mathrm{A}, \mathrm{B}}(j, k)$ that an object in a magnitude bin $j$ is recovered in a bin $k$ where $\mathrm{A}$ or $\mathrm{B}$ refers to one of the half-catalogs. The completeness is then $C_{\mathrm{A}}(j)=$ $\sum_{k} P_{\mathrm{A}}(j, k)$ and similarly for image B. Let $N_{R}(j)$ be the number of real objects whose true magnitudes lie within bin $j$. In addition, due to noise fluctuations (both Poisson and nonrandom), there will be some false objects detected in bin $j$. Let $f_{\mathbf{A}}(j)$ be the probability that any object in image $\mathbf{A}$ in bin $j$ is a false detection, and let $N_{\mathbf{A}}(j)$ be the number of objects found in bin $j$ on image A. Then

$$
N_{\mathbf{A}}(j)=\sum_{k} N_{R}(k) \times P_{\mathbf{A}}(k, j)+f_{\mathbf{A}}(j) \times N_{\mathbf{A}}(j) ;
$$

and

$$
N_{\mathrm{B}}(j)=\sum_{k} N_{R}(k) \times P_{\mathrm{B}}(k, j)+f_{\mathrm{B}}(j) \times N_{\mathrm{B}}(j) .
$$

We then count the number of objects $N_{\mathrm{AB}}(i, j)$ that appear in common in both half-images A and B (i.e., which agree in position to within 0 ". 25 of each other) and that have measured magnitudes in A that place them in bin $i$ and measured magnitudes in $\mathrm{B}$ that place them in bin $j$. Then

$$
N_{\mathrm{AB}}(i, j)=\sum_{k} N_{R}(k) \times P_{\mathrm{A}}(k, i) \times P_{\mathrm{B}}(k, j) .
$$

Strictly speaking, we should add to equation (7) a term that represents the number of times that a false detection in both half-images will be coincident to within 0 ".25 and will land in the two magnitude bins in question. In practice this number is small compared to one, and we neglect it.

If $N_{\text {bins }}$ is the number of magnitude bins, then equations (5) and (6) hold for the $N_{\text {bins }}$ values of $j$ and in equation (7) for the $N_{\text {bins }} \times N_{\text {bins }}$ combinations of $i$ and $j$. The unknown quantities are the $N_{\text {bins }}$ values for the number of real sources with true flux placing them in bin $N_{R}(k)$ along with the $N_{\text {bins }}$ estimates for the probabilities $f_{\mathrm{A}}(j)$ and $f_{\mathrm{B}}(j)$ that a given source in bin $j$ is not a real source. Obviously, the system is overdetermined. This is to be expected since equations (5), (6), and (7) are just discrete representations of integral equations describing the observed number count distribution from which we are trying to recover the true distribution, taking into account losses, false sources, and errors between the true and measured magnitudes due to noise.

A simplification of the preceding equations that is useful for illustrative purposes and actual calculations in some cases comes from ignoring the cross-terms and letting the completeness in any bin be equal to $C$ independent of which half-catalog is addressed. This eliminates the cross terms in equations (5), (6), and (7).

In that case, we obtain for each bin,

$$
N_{R}=N_{\mathrm{AB}} / C^{2}
$$

and

$$
f_{\mathrm{A}}=1-N_{\mathrm{AB}} /\left(C \times N_{\mathrm{A}}\right)
$$

with a similar expression for $f_{\mathrm{B}}$.

$$
\text { 10.1.2. Negative Image Tests }
$$

As described below, we are limited in the applicability of the full formalism described above owing to small number 
statistics in the observed number of objects, which can lead to reliability estimates greater than 1 . An alternative procedure is to multiply the final images by -1.0 and search for "detections" of objects in these negative images. This assumes that the noise properties of the images are the same for negative excursions as for positive ones. This is not in general true since, for example, cosmic rays that are not completely removed have no counterpart in the negative image. In the case of the NICMOS HDF images, however, not only are the cosmic rays removed fairly effectively within each frame as a consequence of the nondestructive readout but the very large number of dithered frames making up our final images also reduces any residual cosmic rays by a further larger factor. Unfortunately, we have found that this method does not appear to be well suited for the KFOCAS extractions for reasons associated with edge effects near the large negative "holes" in the counts produced by the bright real sources in the negative images. However, this method does seem to yield useful results for the SExtractor algorithm. We now discuss the particular tests actually carried out on the KFOCAS and SExtractor half-catalogs.

\subsection{KFOCAS Half-Catalog Tests}

The KFOCAS analysis of each half-image used the same parameters as the total image analysis. Since the images are in units of photon flux, the half-images have the same signal strength for true sources but have a higher noise. Unlike SExtractor, the $1 \sigma$ noise level for KFOCAS is an input parameter. Retention of whole catalog input parameters results in an input $1 \sigma$ noise value that is a $\sqrt{2}$ lower than the noise in the half-catalog. The half-image KFOCAS analysis then detects more sources, since more random noise fluctuations appear above the detection threshold. Bright true sources should be detected in both images. Faint sources of course could be detected in only one or even neither of the half-images. Each source is marked in the catalog test column as to whether it appeared in both, only one, or none of the half-images.

In practice for the KFOCAS source we utilize the simplified formalism described in equations (8) and (9) of $\S$ 10.1.1. In particular we note that the reliability in either halfcatalog is $1-f_{\mathrm{A}}$ or $1-f_{\mathrm{B}}$, so we can say that the reliability $r$ is

$$
r_{\mathrm{A}}=N_{\mathrm{AB}} /\left(C \times N_{\mathrm{A}}\right) .
$$

Since all of the quantities on the right-hand side of equation (10) are known, $r$ can be calculated using the values of $C$ previously determined. However, when this is formally carried out, the values of $r$ for some magnitude bins become greater than 1 owing to a low value of $C$ for that bin or small number statistics. Since the true value of the completeness can never exceed one we can get a robust lower limit on $r$ by setting $C$ equal to 1 and noting that

$$
r_{\mathrm{A}} \geq N_{\mathrm{AB}} / N_{\mathrm{A}}
$$

with again a similar equation for $r_{\mathrm{B}}$. This equation depends solely on the ratio of the number of sources detected in both catalogs to the number detected in one of the half-catalogs. The final reliability for a magnitude bin is just the average of $f_{\mathrm{A}}$ and $f_{\mathrm{B}}$. This reliability is appropriate for the halfcatalogs. The signal-to-noise ratio in the whole catalog is a factor of $\sqrt{2}$ higher. This corresponds the objects in the half-catalog that are the same factor brighter. This is an offset of $0.376 \mathrm{mag}$; therefore, the calculated reliability numbers in the half-catalog are appropriate for sources that are 0.376 mag fainter in the whole catalog.

Table 5 contains the results of these tests under the section marked KFOCAS. The completeness values listed in the table are the values found from the analysis in $\S 9.1$. The reliability numbers are the numbers from the above calculation adjusted to the appropriate aperture magnitude for the total catalog. These values are in general lower than those calculated using the measured values for completeness in equation (10).

\subsection{Sextractor Half-Catalog Tests}

For both the whole image and half-images, we use the SExtractor parameters given in Table 3 but determine the completeness independently for the half-images using the same procedure described in $\S 9$.

As noted above, the noise properties in the half-images scale almost exactly as expected, so that the false detection rate at a given magnitude bin in the half-images should be applied to a magnitude bin in the whole image fainter by $1 / \sqrt{2}$ lower in the flux, or 0.376 mag. We then use the mean of the two false detection rates determined from the halfimages for the estimate of the false detection rate at this slightly fainter magnitude.

As described in $\S 10.1 .1$, the system of equations (5)-(7) for $N_{R}(k)$ is overdetermined, and we determined these values by a least-squares fit to the observed values $N_{\mathrm{AB}}(i, j)$. Equations (5) and (6) then give the false detection rates for the two half-images and use the mean of these determinations for the whole image as explained above.

In practice, as already discussed in $\S 10.2$, the small number of sources actually detected in common in the two half-images results in uncertainties in the reliability estimates for magnitudes at which the completeness is near unity. We have also estimated the reliability of the detections by the negative image method described in $\S 10.1 .2$. The objects that SExtractor finds using this technique do not seem to occur preferentially near the "holes" associated with the negative sources but occur in the higher signal-tonoise ratio regions, as expected, so that SExtractor does not seem subject to the same degree to the edge effects described in $\S 10.1 .2$ for KFOCAS.

\subsection{The Completeness and Reliability Table}

The completeness and reliability table, Table 5, summarizes the results of our tests described above. Columns (2)-(8) refer to the KFOCAS reductions only, and columns (9)-(11) refer to the results from SExtractor. As described in $\S 7.4$, there is a systematic difference between aperture magnitudes measured by KFOCAS and SExtractor, which becomes significant for objects fainter than $\approx 28.0$, as shown in Figure 8. Thus, in the first column, the magnitude is the aperture $\mathrm{AB}$ magnitude measured by KFOCAS for the KFOCAS reductions and by SExtractor for the SExtractor reductions. The width of the magnitude bin is $0.5 \mathrm{mag}$ centered on the value in the magnitude column. The signal to noise ratio $(\mathrm{S} / \mathrm{N})$ is the average signal-to-noise ratio for all objects in the magnitude bin. The columns labeled $C s_{16}$, $\mathrm{Cl}_{16}, \mathrm{Cs}_{11}$, and $C \mathrm{Cl}_{11}$ are the completeness numbers for the KFOCAS reduction listed in order for small and large objects in the F160W filter and the F110W filter, respectively. Next are the reliability numbers for the F160W and 
F110W filters, where no discrimination has been made between small and large objects. Completeness and reliability SExtractor results for the F160W filter comprise the last three columns, where the column labeled $R_{\mathrm{co} 16}$ uses the full half-image formalism, while the column labeled $R_{\text {neg } 16}$ uses the negative image technique. It should be emphasized that the reliability estimates at the faint end of the table are subject to considerable uncertainty. However, the results all seem to indicate a fairly low incidence of false detections $\sim 5 \%-15 \%$ at magnitude $\sim 27.5$, but this incidence rises steeply at fainter magnitudes, while the completeness is of order $\sim 80 \%-90 \%$ at magnitude 27.5 , of order $\sim 70 \%-75 \%$ at magnitude 28.0, and falls rapidly beyond that point. KFOCAS appears to lose more objects owing to merging with other objects than SExtractor particularly for bright objects. This is probably the cause of the less than $100 \%$ completeness at bright magnitudes for the KFOCAS reductions. The low number of objects in the brighter bins limits the accuracy of the measurements, and differences of $\pm 5 \%$ should not be considered significant.

Our discussion of the differences in source extractions in $\S 7.4$ clearly indicates that the IDL and IRAF images are essentially identical and that the magnitude differences in Figure 8 are due solely to differences between the two extraction programs, KFOCAS and SExtractor. There are also differences in the number of detections between the two programs. Running SExtractor on the IDL image with the same set of parameters used for the SExtractor analysis of the IRAF images, we find 284 galaxies, somewhat less than the 356 found on the IRAF image. On the other hand, there are a total of 350 objects selected by KFOCAS from the whole IDL F160W image, also more than those found by SExtractor on the IDL image. The total number of objects found in the F160W image by KFOCAS and in the IRAF image by SExtractor is nearly identical.

Inspection of Table 5 indicates the range of reliability and completeness measures returned by the two methods. In general the reliability and completeness measures from the KFOCAS analysis fall below those determined via the SExtractor analysis. This is particularly true when one considers the difference in faint magnitudes discussed in $\S 7.4$. This indicates that at the faintest magnitudes the KFOCAS numbers should be compared with the SExtractor numbers for sources with SExtractor magnitudes nearly a magnitude brighter than the KFOCAS magnitude. Part of this difference in reliability is due to the KFOCAS numbers being lower limits on the reliability as discussed in $\S 10.2$. Another part of the difference, however, is due to the uncertainty inherent in these calculations, and users of this catalog should be aware of them. Our net philosophy is to be aggressive in identifying potential sources but to be relatively conservative in calculating their reliability and completeness.

\section{GALAXY COUNTS}

As with the original optical catalog of Williams et al. (1996), it is not our intention in this paper to discuss scientific results. A commonly used statistic, however, is the differential number count of galaxies. Figure 10 presents this statistic for the region of the HDF covered by our catalog. The galaxy counts in number per magnitude per square degree have been divided into half-magnitude bins. If the object radius is less than 0.3 , the aperture magnitude is used. If the object radius is greater than this, the isophotal

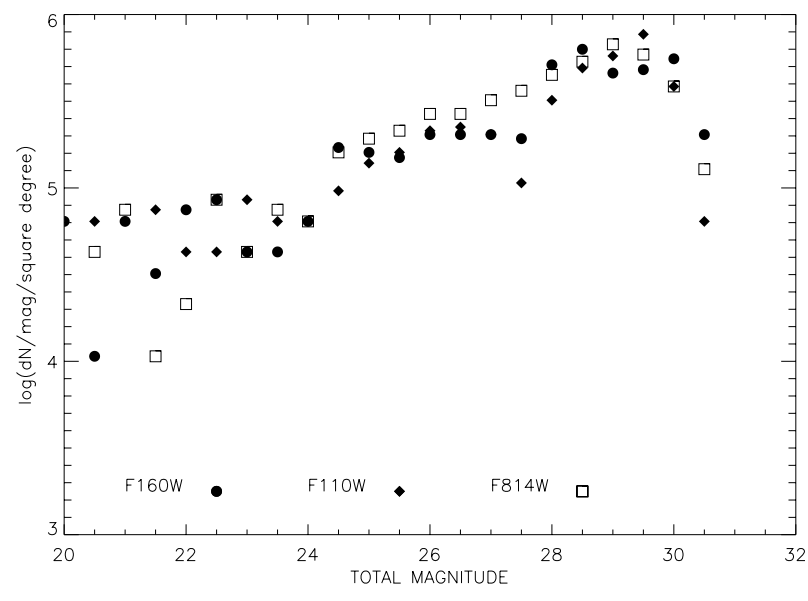

FIG. 10.-Differential galaxy counts as a function of $A B$ aperture magnitude only of the sources appearing in the sigma-limited catalog.

magnitude is used. The number counts for the F814W filter in the same area as determined from the optical catalog are also shown for comparison. The same division between aperture and isophotal magnitudes are used for the F814W data. The counts for the WFPC2 F814W objects includes all of the WFPC2 catalog objects in the NICMOS field, not just those in common with NICMOS objects. There are no aperture corrections applied to this data in order to facilitate comparison with the statistics presented in Williams et al. (1996). It should be noted that the area covered by the NICMOS image is very small. For a value of $H_{0}=50$ and a value of $\Omega_{0}=1$, the sides of the NICMOS image are on the order of $250 \mathrm{kpc}$ for redshifts greater than 1 . This is about 10 times smaller that the typical diameter of a region forming a single galaxy from cold dark latter simulations (Steinmetz 1998). Drawing any cosmological conclusions from this small sample may be very suspect. Following the discussion in Williams et al. (1996) we have not eliminated split objects from the number counts. Except at very bright magnitudes, we do not expect this to affect the statistics significantly.

\section{CONCLUSIONS}

The NICMOS observations of the Hubble Deep Field add significant value to the existing data by providing improved wavelength coverage and access to objects that are either too heavily extincted or too highly redshifted to be visible in the original optical catalog. This paper is designed to be a reference source for the use of this data in various areas of research. Future papers will discuss various aspects of the significance of these data.

Many people contributed to the NICMOS observations of the Hubble Deep Field. The entire NICMOS Instrument Definition Team contributed to the success of NICMOS and participated in the decision to allocate a large fraction of the team's guaranteed time to this effort. We wish to thank Mark Dickinson for help with the KFOCAS reduction techniques and Andy Fruchter for his aid in implementing the Drizzle image procedure. Andy Lubenow spent many hours refining our observation plan to handle the single guide star acquisition. Chris Conner and the Lockheed MOSES group went to extraordinary efforts to 
keep the gyro biases updated to ensure good pointing under single guide star tracking. Zolt Levay provided invaluable assistance in the preparation of the images for publication and E. Bertin provided the SExtractor software and quick response to inquiries. The schedulers at STScI diligently worked to minimize the impact of SAA crossings. L. S.-L. thanks Lin Yan and Patrick McCarthy and R. J. W. thanks
David Koo for very useful discussions on incompleteness testing and galaxy surveys. This work was supported by NASA grant NAG 5-3043, and the observations were obtained with the NASA/ESA Hubble Space Telescope operated by the Space Telescope Science Institute managed by the Association of Universities for Research in Astronomy Inc. under NASA contract NAS5-26555.
Bertin, E., \& Arnouts, S. 1996, A\&A, 117, 393

Connolly, A. J., Szalay, A. S., Dickinson, M., SubbaRao, M. U., \& Brunner, R. J. 1997, ApJ, 486, L11

Dickinson, M., et al. 1997, HST Approved Cycle 7 Observing Plan

Fruchter, A. S., \& Hook, R. N. 1997, Proc. SPIE, 3164, 120

Fruchter, A., Hook, R. N., Busko, C., \& Mutchler, M. 1997, in The 1997 HST Calibration Workshop, ed. S. Casertano, R. Jedrzejewski, C. D. Keyes, \& M. Stevens (Baltimore, MD: STScI), 518

Jarvis, J. F., \& Tyson, J. A. 1981, AJ, 86, 476

Lehar, J., Falco, E. E., Impey, C. D., Kochanek, C. S., McLeod, B. A., Munoz, J., \& Rix, H.-W. 1999, in preparation

McLeod, B. 1997, in The 1997 HST Calibration Workshop, ed. S. Casertano, R. Jedrzejewski, C. D. Keyes, \& M. Stevens (Baltimore, MD: STScI), 281

\section{REFERENCES}

Pozzetti, L., Madau, P., Zamorani, G., Ferguson, H. C., \& Bruzual A., G. 1998, MNRAS, 298, 1133

Steinmetz, M. 1998, in Space Telescope Symposium on the Hubble Deep Field, in press

Valdes, F. 1982, Faint Object Classification and Analysis System (KPNO Internal Publication)

Williams, R. E., et al. 1996, AJ, 112, 1335.

Yan, L., McCarthy, P. J., Storrie-Lombardi, L. J., \& Weymann, R. J. 1998, ApJ, 503, L19 